\title{
CFD optimisation of a stadium roof geometry: a qualitative study to improve the wind microenvironment
}

\author{
Polytimi Sofotasiou ${ }^{1}$, Ben Hughes ${ }^{1, *}$, and Saud Abdul Ghani ${ }^{2}$ \\ ${ }^{1}$ Department of Mechanical Engineering, University of Sheffield, Sheffield, UK \\ ${ }^{2}$ Department of Mechanical and Industrial Engineering, Qatar University, Doha, Qatar
}

Received: 16 June 2017 / Accepted: 3 July 2017

\begin{abstract}
The complexity of the built environment requires the adoption of coupled techniques to predict the flow phenomena and provide optimum design solutions. In this study, coupled computational fluid dynamics (CFD) and response surface methodology (RSM) optimisation tools are employed to investigate the parameters that determine the wind comfort in a two-dimensional stadium model, by optimising the roof geometry. The roof height, width and length are evaluated against the flow homogeneity at the spectator terraces and the playing field area, the roof flow rate and the average interior pressure. Based on non-parametric regression analysis, both symmetric and asymmetric configurations are considered for optimisation. The optimum design solutions revealed that it is achievable to provide an improved wind environment in both playing field area and spectator terraces, giving a further insight on the interrelations of the parameters involved. Considering the limitations of conducting a twodimensional study, the obtained results may beneficially be used as a basis for the optimisation of a complex threedimensional stadium structure and thus become an important design guide for stadium structures.
\end{abstract}

Keywords: computational fluid dynamics / response surface methodology / optimisation / wind comfort / stadiums

\section{Introduction}

Stadium structures demand particular care during design process to comply with the manufacturing and environmental standards, due to the large number of attendees that they tend to attract. For this reason, progressive design strategies are ordinarily deployed in conceptual phase, in order to combine all the requisite components that will advocate for the optimality of the constructional result. The optimisation of every design should mainly focus on the improvement of users' comfort within the building environment, while aiming at the harmonious coexistence of an environmentally susceptible and climate rational design [1].

This study is focused on the design optimisation of the roof geometry of a two-dimensional (2D) stadium model using coupled computational fluid dynamics and response surface methodology optimisation techniques. Based on rational design interventions on the roof configuration, the work pursues the provision of improved or optimum microclimate conditions in the arena bowl, including both spectator terraces and playing field area. The qualitative study includes the exploration of both symmetric and

* e-mail: ben.hughes@sheffield.ac.uk asymmetric roof alterations and the corresponding analytical results on velocity measurements and flow homogeneity at the areas of interest.

\section{Previous related work}

Early studies on stadium structures highlighted the multidimensional and multiscalar characteristics of thermal comfort and energy consumption in stadiums and accentuated the need to adopt coupled simulation software techniques to obtain a holistic design approach $[2,3]$. Since then, several studies have been performed to determine the structural characteristics that contribute the most to the alteration of the microenvironment at the stadium bowl.

CFD studies validated with wind tunnel experiments have shown that the roof configuration and the porosity of the stadium envelop have great impact on the wind distribution at both pitch level and spectator terraces and consequently on the induced wind comfort conditions [4-7]. Additional studies revealed the importance of providing ventilation openings to promote natural airflow and ventilation at the occupied areas of the stadium bowl $[6,8,9]$. The interactions between the urban environment and the stadium structures as well as the wind comfort conditions on pedestrian level have also attracted great 
Table 1. Four-step methodology of the RSM optimisation study [19].

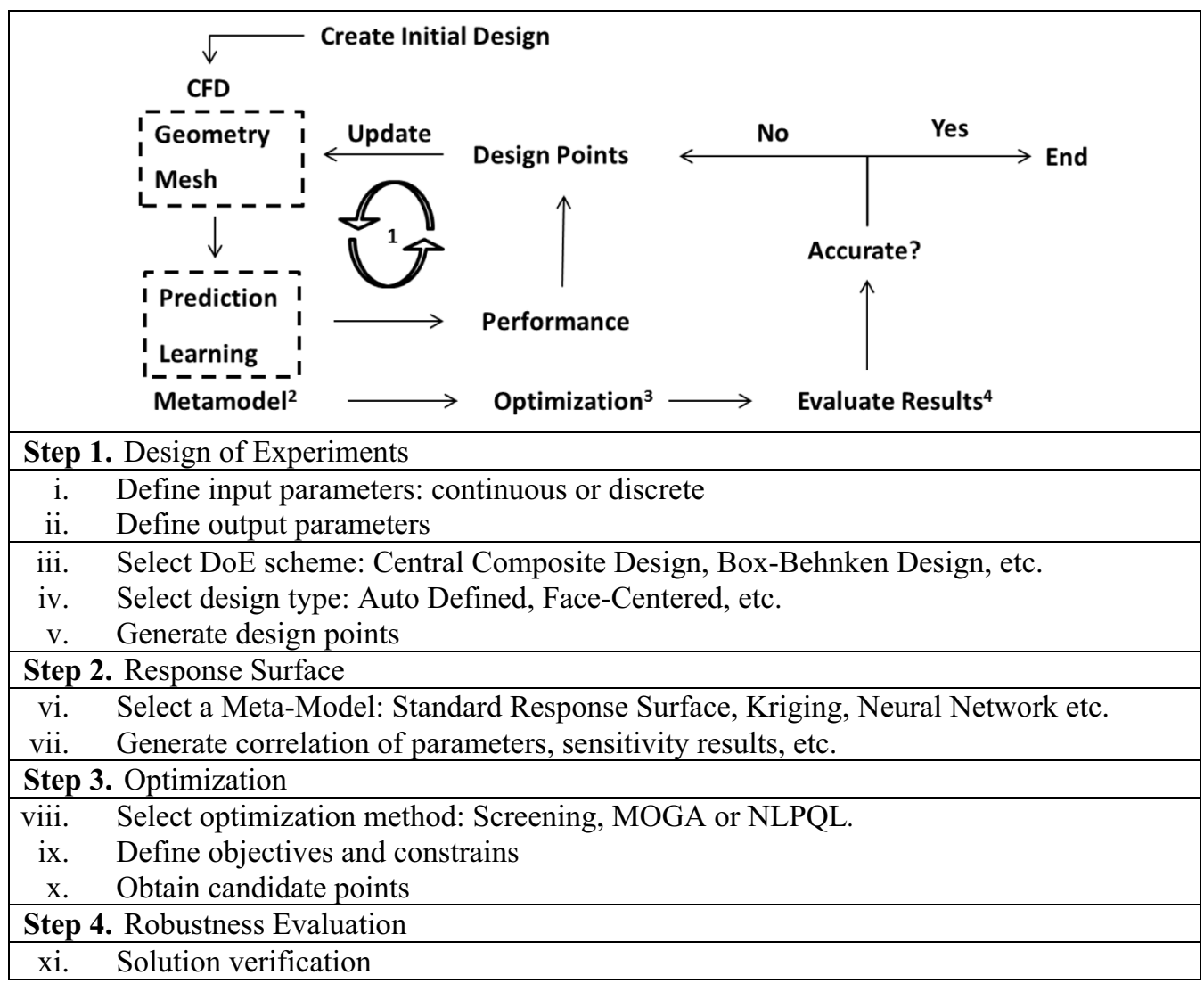

interest [10-12]. Finally, thermal comfort studies, employing dynamic thermal modelling and CFD techniques, were also conducted to assess thermal discomfort and temperature distribution on stadia $[2,13-15,41]$ and energy consumption requirements $[16,17,42]$.

To date, only few of the aforementioned research works have been produced with intention to provide a design solution with improved responses towards wind comfort. The completed parametric studies have been conducted under limitations of design parameters and experimental variations. In this study, the design exploration of various roof configurations, within a predefined two-dimensional design space, is pursued to provide improved wind environmental conditions and understand the interrelationships of the design parameters involved. To the authors' knowledge, no previous work has used combined CFD and RSM methods to assess and optimise a stadium or its components. This study will detail the method used, which will be useful for optimising stadium structures, since the method can be replicated for the optimisation of any roof and also other structural components.

\section{Methodology}

For the purpose of this study, coupled CFD modelling and RSM optimisation techniques were employed. CFD is a widely recognised and well-documented simulation tool that employs numerical methods and algorithms to produce highly accurate predictions of flow movement and distribution inside and outside building structures [18]. On the other hand, RSM is a meta-modeling-based optimisation technique that seeks local and/or global optimality, via the evaluation of the assigned design parameters. With substantially limited investment in computational cost, it provides the opportunity to evaluate numerous design cases and assess the influential role of the designated factors.

The simulation-based optimisation study was conducted in four main steps. A 2D stadium design was initially evaluated against velocity and pressure distributions. A series of sequential simulation runs was then performed generating data, regarding predefined geometrical characteristics of the stadium roof (Step 1). The results were used to formulate a high precision mathematical model that would describe the problem and predict relationships among variables (Step 2). The optimisation process was performed according to the selected algorithm and the defined objective sets and thresholds, pursuing design optimality (Step 3). Finally, the proposed solutions were verified with simulation runs and compared with the predicted ones, in order to evaluate the solution and therefore the accuracy of the RSM-based optimisation (Step 4). The flowchart in Table 1 is a graphical representation of the steps followed for the optimumseeking stadium roof configuration to create improved microenvironmental conditions. The methodology followed 

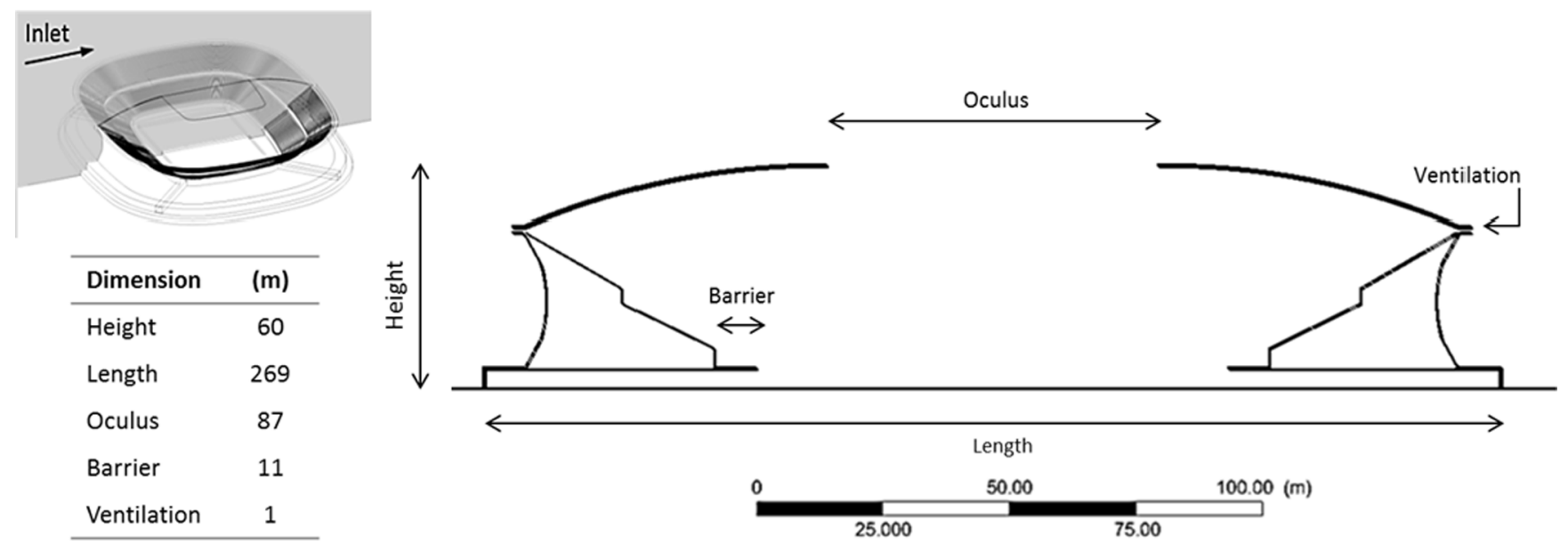

Fig. 1. Dimensional characteristics of the 2D stadium model.

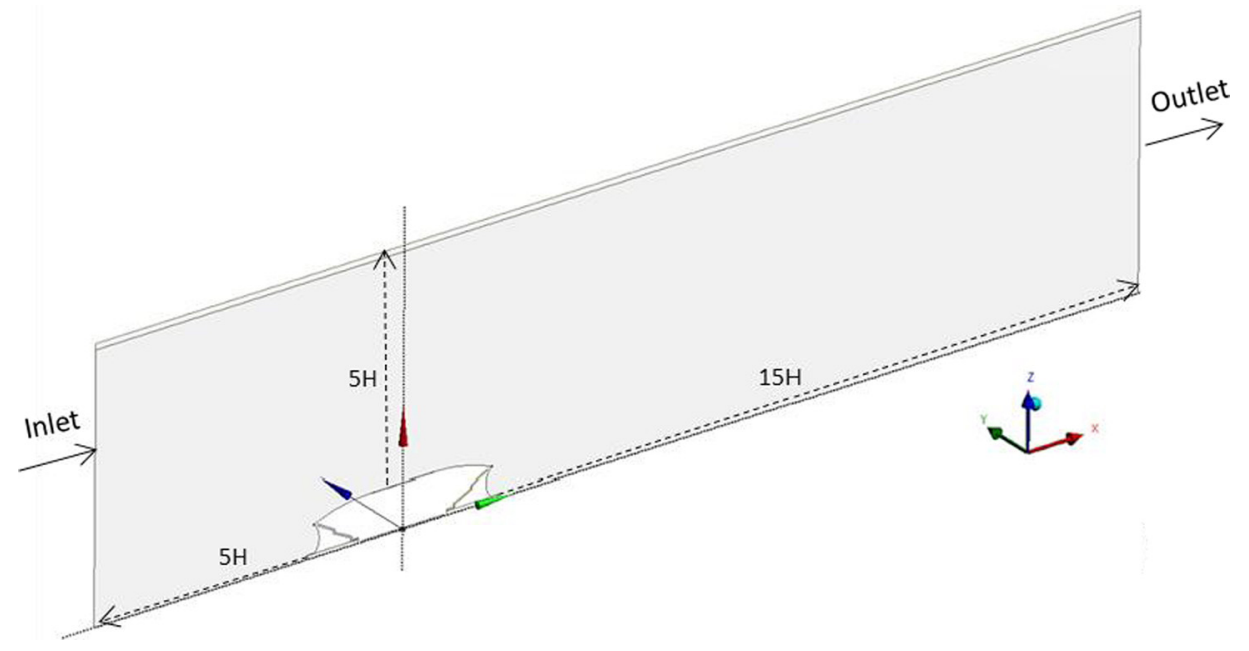

Fig. 2. Dimensional characteristics of the $2 \mathrm{D}$ computational domain.

is fully detailed and validated [19] and it is a computational inexpensive solution to provide quantitative and qualitative results of improved design solutions.

\section{Case study description}

The 2D stadium design was based on FIFA's technical requirements and recommendations for international games, with a seating capacity of 60000 [20]. The dimensional characteristics indicate a structure of $60 \times 269 \mathrm{~m}^{2}(H \times L)$. The oculus roof configuration is represented by a $95 \mathrm{~m}$ opening, long enough to provide adequate sunlight for the natural grass growth. The stadium consists of two spectator terraces, which are fully covered by the roof configuration, to reduce the direct solar irradiance (Fig. 1). They are also placed within a distance of $11 \mathrm{~m}$ far from the playing field to lessen the impact of the expected central vortex in the stadium bowl [5]. Furthermore, a ventilation opening of $1 \mathrm{~m}$ was created between the roof and the upper spectator tiers to allow for natural ventilation [6].

\section{CFD settings and parameterisation}

For the simulation of the wind distribution, the standard $k-\varepsilon$ turbulence model was used, which is accepted and validated for $2 \mathrm{D}$ case studies [21-24].

\subsection{Geometry and mesh generation}

Regardless of the two-dimensional geometry, the recommendations for CFD simulations for urban wind studies were followed [25]. Given the height of the stadium structure $(H=60 \mathrm{~m})$, the computational domain was spanning $300 \mathrm{~m}(5 H)$ upstream, $900 \mathrm{~m}(15 H)$ downstream and $360 \mathrm{~m}(6 H)$ on the vertical direction. Thus, the modelled domain would allow for fully developed and homogeneous ABL flow prior approaching the stadium structure, as depicted in Figure 2.

The complexity of the geometry and the large difference in length scales complicated the generation of a fully structured mesh. The high-resolution mesh close to the stadium walls, the ground walls and the ventilation 

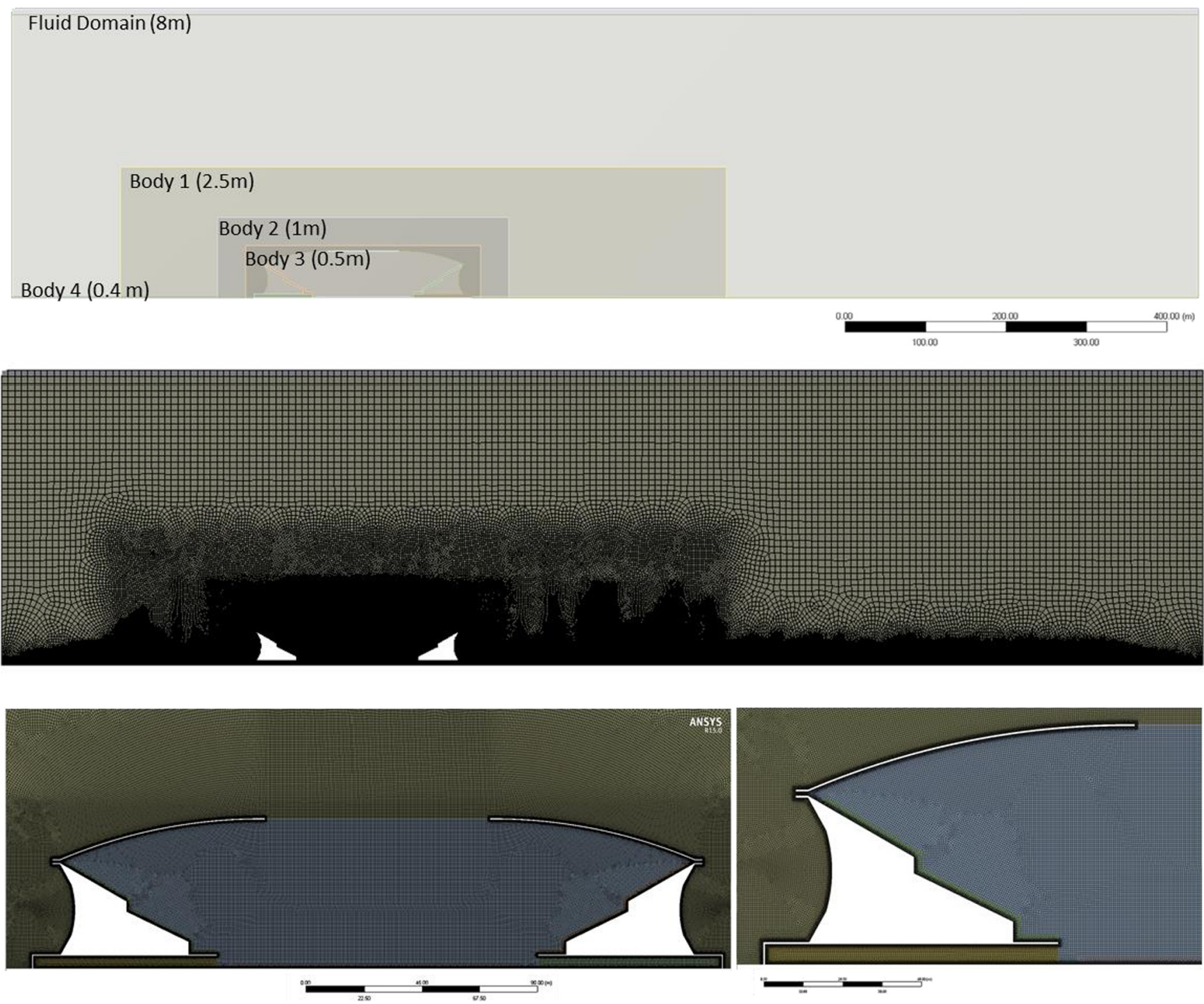

Fig. 3. Creation of bodies of influence and generation of controlled meshing size.

openings was achieved by local size control on bodies of influence. As shown in Figure 3, four bodies of influence were created to facilitate the orderly evolution from coarser mesh in the outer domain to a finer mesh in the stadium area. Starting with a cell size of $8 \mathrm{~m}$ in the outer domain, the mesh was reduced to $2.5 \mathrm{~m}$ cell size for Body 1 , $1 \mathrm{~m}$ for Body 2 and $0.5 \mathrm{~m}$ for Body 3 that included the area on the exact proximity of the stadium. Body 4 included the ground area with a cell size of $0.4 \mathrm{~m}$. On the stadium walls a grid refinement control of 3 was applied. This method ensured the smooth transition from the wind urban scale to the building scale. The hybrid mesh consisted of 237703 quadrilateral and triangular cells.

\subsection{Grid verification}

Three different mesh sizes were tested to ensure mesh independency of the solution. The initial mesh consisted of 237703 cells, the coarser mesh with 122709 cells and the finer one with 576620 hybrid cells. The selection of the favourable mesh size relied on the correlation of the dimensionless values of velocity $\left(U / U_{\text {free-stream }}\right)$ and the longitudinal direction of the flow $\left(x / L_{\text {Stadium }}\right)$, along a line passing through the ventilation openings. The correlation of the resultant values is presented in Figure 4 and show good prediction for all three meshes.

Additional comparative results were generated for the flow rate at the front ventilation opening. As presented in Table 2 , the maximum error of $5.4 \%$ was calculated for the coarse grid size, as opposed to $1.8 \%$ for the fine mesh. Thus, the medium mesh was selected for the current simulation study, allowing computational-time savings and a high degree of solution accuracy.

\subsection{Boundary conditions}

The simulation of the wind distribution includes the generation of an atmospheric boundary layer (ABL). The mean ABL velocity profile follows a logarithmic production 


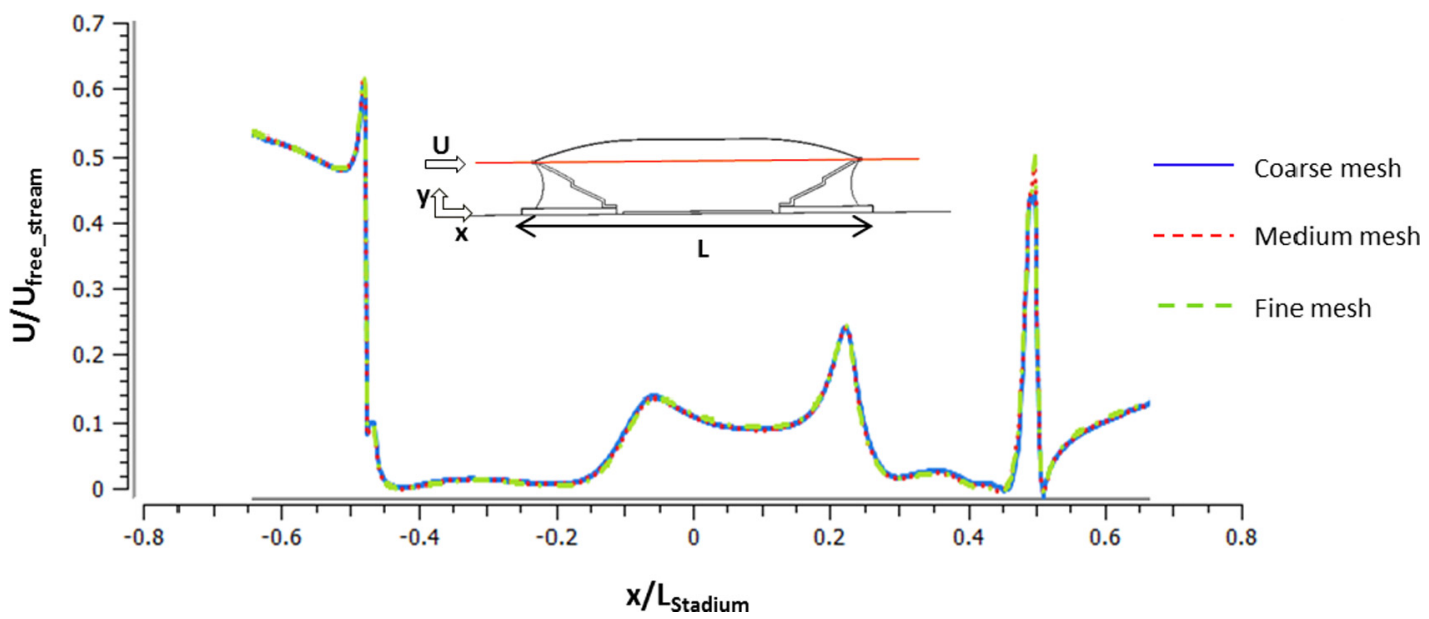

Fig. 4. Grid sensitivity study among the three different mesh sizes; coarse mesh (122 709), medium mesh (237 703), fine mesh $(576620)$.

Table 2. Flow rate error for the three grid sizes.

\begin{tabular}{lll}
\hline $\begin{array}{l}\text { Computational } \\
\text { grid size }\end{array}$ & $\begin{array}{l}\mathrm{Q}_{-} \text {flow rate } \\
\left(\mathrm{m}^{3} / \mathrm{s}\right)\end{array}$ & $\varepsilon=\left(f_{2}-f_{1}\right) / f_{1} \times 100 \%$ \\
\hline Coarse: 122709 & $-0.5394^{*}$ & $5.4 \%$ \\
Middle: 237703 & $-0.5115^{*}$ & - \\
Fine: 576620 & $-0.5208^{*}$ & $1.8 \%$ \\
\hline
\end{tabular}

* The negative sign indicates that the flow exits the stadium interior from the ventilation opening.

that when it is fully developed it can be described by equation (1) suggested by [26], followed by the mean turbulent kinetic energy (2) and dissipation rate profiles (3):

$$
\begin{gathered}
u=\frac{u_{\mathrm{ABL}}^{*}}{\kappa} \ln \left(\frac{y+y_{0}}{y_{0}}\right), \\
k=\frac{u_{\mathrm{ABL}}^{* 2}}{\sqrt{C_{\mu}}} \\
\varepsilon=\frac{u_{\mathrm{ABL}}^{* 3}}{\kappa\left(y+y_{0}\right)} .
\end{gathered}
$$

where $u_{\mathrm{ABL}}^{*}$ is the ABL friction velocity equal to $0.77 \mathrm{~m} / \mathrm{s}$ calculated from the reference velocity $U_{\text {ref }}(7 \mathrm{~m} / \mathrm{s})$ at reference height $y_{\text {ref }}(22 \mathrm{~m})$, obtained from the meteorological station of the Al-Khor city in Qatar, $y_{0}$ is the aerodynamic roughness length $(0.5 \mathrm{~m}), \kappa$ is the von Korman constant equal to 0.42 , and $C_{\mu}$ is an empirical derived constant, equal to 0.09 in the standard $k-\varepsilon$ model.

For the development of the logarithmic inlet velocity profile a user-defined function (UDF) was produced based on equations (1)-(3). It was also decided to improve the case model by applying constant shear stress on the top row of cells equal to $\tau_{w}=\rho u_{*}^{2}[26]$, which is also suggested by [27]. The parameterisation of the computational model is

\begin{tabular}{|c|c|}
\hline Turbulent model & $\begin{array}{l}\text { Standard } \mathrm{k}-\varepsilon \text { model } \\
\text { with standard wall functions }\end{array}$ \\
\hline \multicolumn{2}{|l|}{ Boundary conditions } \\
\hline Inlet & $\mathrm{UDF}$ for $\mathrm{U}, \mathrm{k}, \varepsilon$ \\
\hline Outlet & $\begin{array}{l}\text { Pressure-outlet with zero } \\
\text { pressure }\end{array}$ \\
\hline Top & Symmetry \\
\hline Ground & $k_{s}=0.2, \mathrm{UDF}$ for $C_{s}$ \\
\hline $\begin{array}{l}\text { Ground-pitch } \\
\text { and stadium } \\
\text { entrances }\end{array}$ & $k_{s}=0.58758, C_{s}=0.5$ \\
\hline \multicolumn{2}{|l|}{ Cell zone conditions } \\
\hline Fluid: top layer & $\begin{array}{l}\text { Source term, } \\
\mathrm{x} \text {-momentum }=0.089 \mathrm{~N} / \mathrm{m}^{3}\end{array}$ \\
\hline Solution methods & $\begin{array}{l}\text { SIMPLE; second order for } \\
\text { pressure, momentum and } \\
\text { turbulence transport equations }\end{array}$ \\
\hline Convergence criterion & $\begin{array}{l}\text { None (to control } \\
\text { end of calculation) }\end{array}$ \\
\hline
\end{tabular}
summarised in Table 3 . The roughness height, $k_{s}$, was
Table 3. Solver settings for the 2D ABL flow.

calculated based on the equation: $k_{s}=9.793 \times y_{0} / C_{s}$. The software does not allow the $k_{s}$ value to be greater than $y_{p}$ that is the distance from the ground to the centre of the first cell. If this is the case, $k_{s}$ is automatically set equal to $y_{p}$. In the current work, the first cell is set equal to $0.4 \mathrm{~m}$ (see Body 4, Fig. 3), resulting in $y_{p}=0.2 \mathrm{~m}$, and thus $k_{s}$ is set equal to $0.2 \mathrm{~m}$. Consequently, the roughness constant $C_{s}$ is greater than 1 , and thus a UDF is required. The playing field area and the stadium entrances have an aerodynamic roughness equal to $0.03 \mathrm{~m}$ (for grass covered area, according to Wieringa [28]), ensuing roughness height equal to $0.58 \mathrm{~m}$, with a set value of $C_{s}$ equal to 0.5 . 


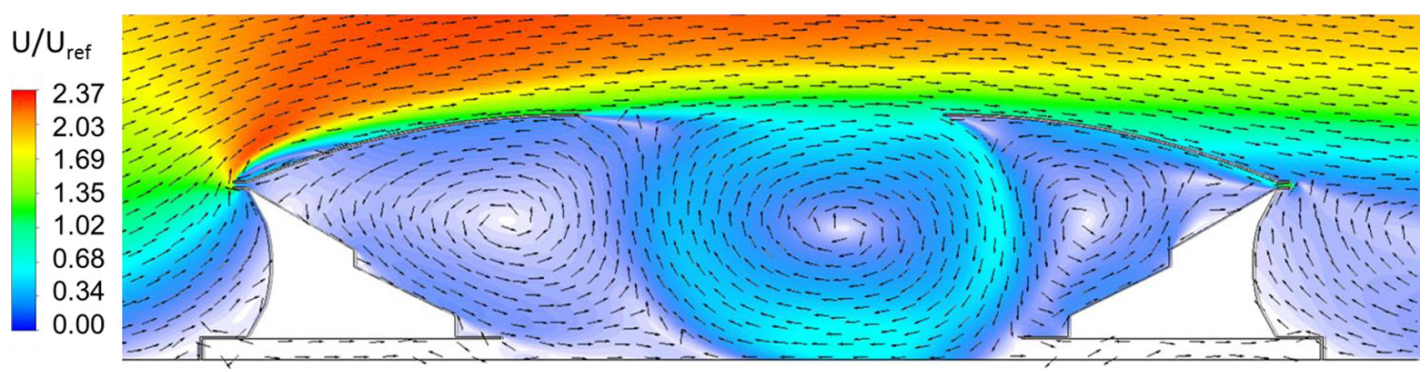

Fig. 5. Normalised velocity vectors and velocity contours of $U / U_{\text {ref, where }} U_{\text {ref }}=7 \mathrm{~m} / \mathrm{s}$ at $22 \mathrm{~m}$ height.

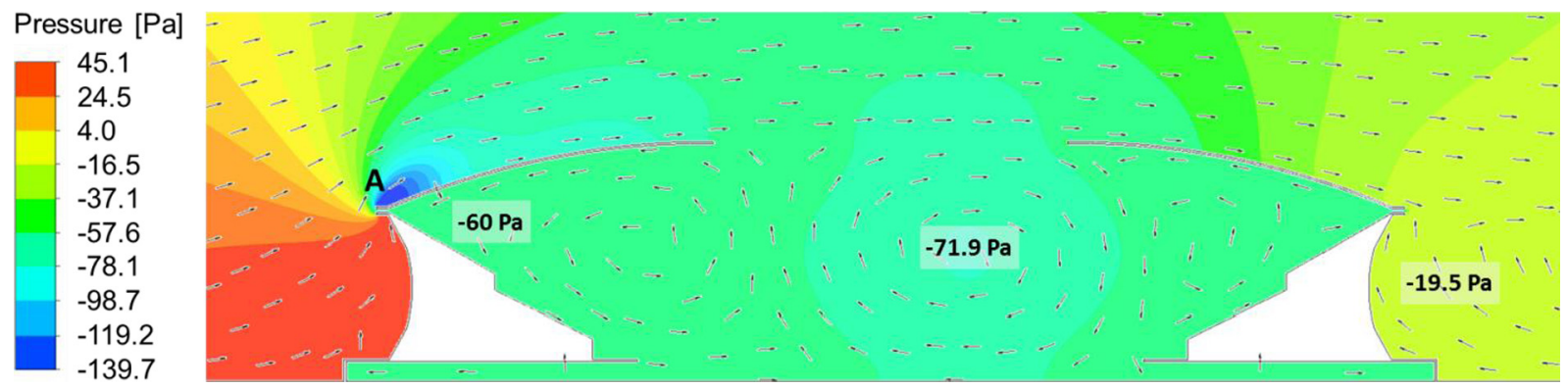

Fig. 6. Pressure contours and velocity vectors for the area outside and inside of the stadium.

\subsection{CFD visualisation}

Building on the initial results of the CFD simulation, velocity and pressure outputs allowed the investigation of: (1) the areas that are mostly influenced by the induced wind,

(2) the wind speed distribution, (3) the wind behaviour and

(4) the potential interventional strategies that could be adopted to improve the stadium's microenvironment. As illustrated in Figure 5, the roof and side ventilation openings seem to have a dominant role on the stadium's microclimate airflow. The incoming air from the roof opening leads to the generation of a primary vortex, located at the centre of the stadium bowl, and clearly shifted towards the leeward terraces. The primary vortex feeds a secondary counterrotating smaller vortex located at the windward spectator tiers, which forces the air to exit the stadium through the front ventilation opening. Additionally, the recirculation zone formed at the rear side of the stadium contributes to the air entrance through the back ventilation opening. Thus, a third vortex is developed, which is spatially limited to the leeward terraces, due to the existence of the primary, and predominant in size, vortex.

The analysis of the pressures acting on the stadium surfaces verifies the wind behaviour at the stadium interior. As shown in Figure 6, the frontal area of the stadium has positive pressure values, as opposed to the rest of the fluid domain. The pressure values at the interior of the stadium vary between $-86.8 \mathrm{~Pa}$ and $-27.5 \mathrm{~Pa}$. The acceleration of the external wind is promoted due to pressure difference, leading to forced directional airflow into the stadium bowl through the roof opening, and thus producing the primary vortex.

In the front of the stadium, positive pressure patterns are observed below the roof level. However, the roof geometry, the extended ventilation opening and the negative angle of attack decelerate the flow leading to adverse pressure gradient and flow separation (Fig. 6, point A). In order to maintain pressure equilibrium, the air exits the stadium via the front ventilation opening. In contrary, on the rear side of the stadium, with pressure values around $-20 \mathrm{~Pa}$, the air is forced to enter the stadium interior via the back ventilation opening.

\subsection{CFD validation}

The validation of the CFD simulation for the $2 \mathrm{D}$ stadium structure was performed based on the study of Persoon et al. [5], who investigated the wind flow patterns and winddriven rain, regarding different roof structures. The development of a primary central vortex is observed for every case study and its size, and/or penetration level under the leeward spectator terraces, is highly dependent on the roof configuration. Secondary vortices on the windward and leeward spectator tiers were also observed in every design case and it was concluded that the size and length of each one of them is subjective to the geometrical characteristics of the stadium itself and the boundary conditions applied (Fig. 7, top).

Similar results were obtained for the current design case. Figure 7 (bottom) illustrates the interaction of the two vortices on the leeward side of the stadium. The primary vortex, created by the air stream entering through the roof opening, extends vertically over the entire height of the stadium bowl. It is well observed that the primary vortex prevails over the secondary one, penetrating about $20 \mathrm{~m}$ below the roof structure (Fig. 7, bottom left). Moving further downstream the roof, the secondary vortex has a more dominant role, directly affecting the wind behaviour on the spectators' level (Fig. 7, bottom right). The rear ventilation opening significantly contributes to the generation of the secondary 
Ascending roof towards the field

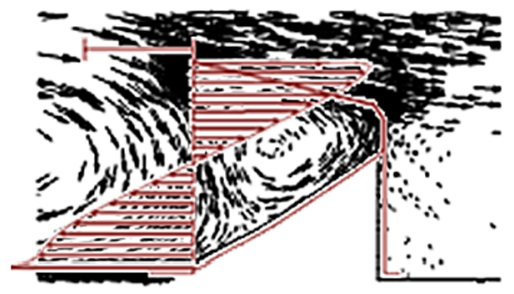

$\mathrm{U}_{\mathrm{u}} / \mathrm{U}_{\mathrm{ref}}=0.2$
Flat roof

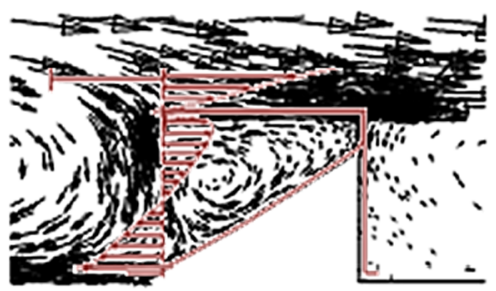

$\mathrm{U}_{\mathrm{u}} / \mathrm{U}_{\mathrm{rer}}=0.2$
Descending roof towards the field

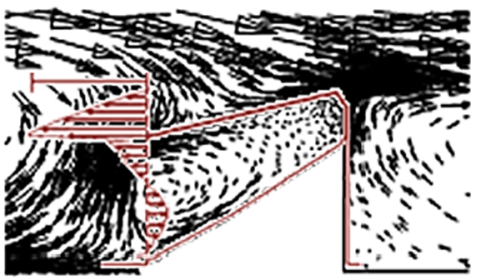

$\mathrm{U}_{\mathrm{u}} / \mathrm{U}_{\mathrm{ref}}=0.2$
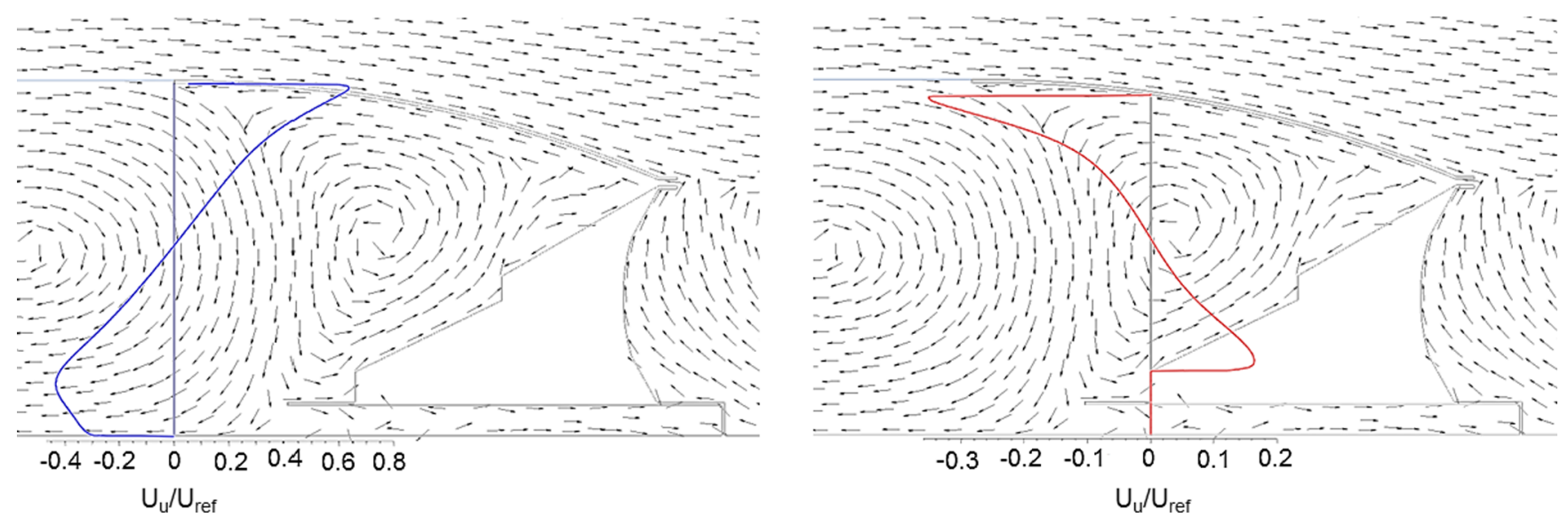

Fig. 7. Wind distribution on three different roof configurations, and corresponding graphs of the dimensionless horizontal component of velocity along the vertical direction [5] (top); interaction between the primary and the secondary vortex, based on the horizontal components of velocity magnitude in two vertical lines (bottom); roof edge level (left) and spectator tiers level (right).

vortex, allowing the pressure difference to naturally move air to the interior. Longwise comparisons of the dimensionless horizontal component of velocity indicate substantially lower velocity magnitudes to be developed by the secondary vortex, as opposed to the primary one.

\section{Design of experiments}

Design of experiments (DoE) is a statistical tool, used to collect and organise data generated from a limited number of experiments. This method enables to quantify how process inputs affect process outputs and study simultaneously several variables in an effort to determine their effects and their interactions. It is the intermediary for the RSM to construct the exploration region of responses, an empirical graph illustrating the response changes, as the continuous variables change under its control.

The three main principles for the implementation of a DoE were described in [29] as follows:

- identify the performance assessment of the dependent variables (outputs) to be optimised, considering the influential role of the most significant independent variables (inputs);

- designate the design space (constrains), within which the input parameters will vary;

- generate the design points and their response values.

\subsection{Selection of input parameters}

According to literature, stadium structures have been extensively studied in order to determine optimum geometrical configurations to provide comfort conditions for both players and spectators [4,5,30]. The roof dimensional characteristics seem to be the major contributors on the attainment of micro-environmental satisfaction. Thus, the input design parameters were set as: (1) the roof height, (2) the roof radius, and (3) the roof length extension towards the centre of the pitch area.

\subsection{Selection of output parameters}

Favourable micro-environmental conditions in semioutdoor stadium structures, initially reported by [30], indicated that aerothermal comfort conditions may be achieved with wind threshold speed values between $0.1 \mathrm{~m} / \mathrm{s}$, to ensure minimum air movement, and $3.6 \mathrm{~m} / \mathrm{s}$, to prevent wind disturbance and performance impairment. On the basis of these considerations, the output parameters were set as the flow homogeneity values on the spectator tiers and the playing field area, the ventilation flow rate through the roof opening, and the overall pressure in the stadium bowl. The maximum and minimum velocity at the occupied areas and the ventilation flow rates through the leeward and windward 
Table 4. Separation of the fluid domain into four controllable zonal areas.
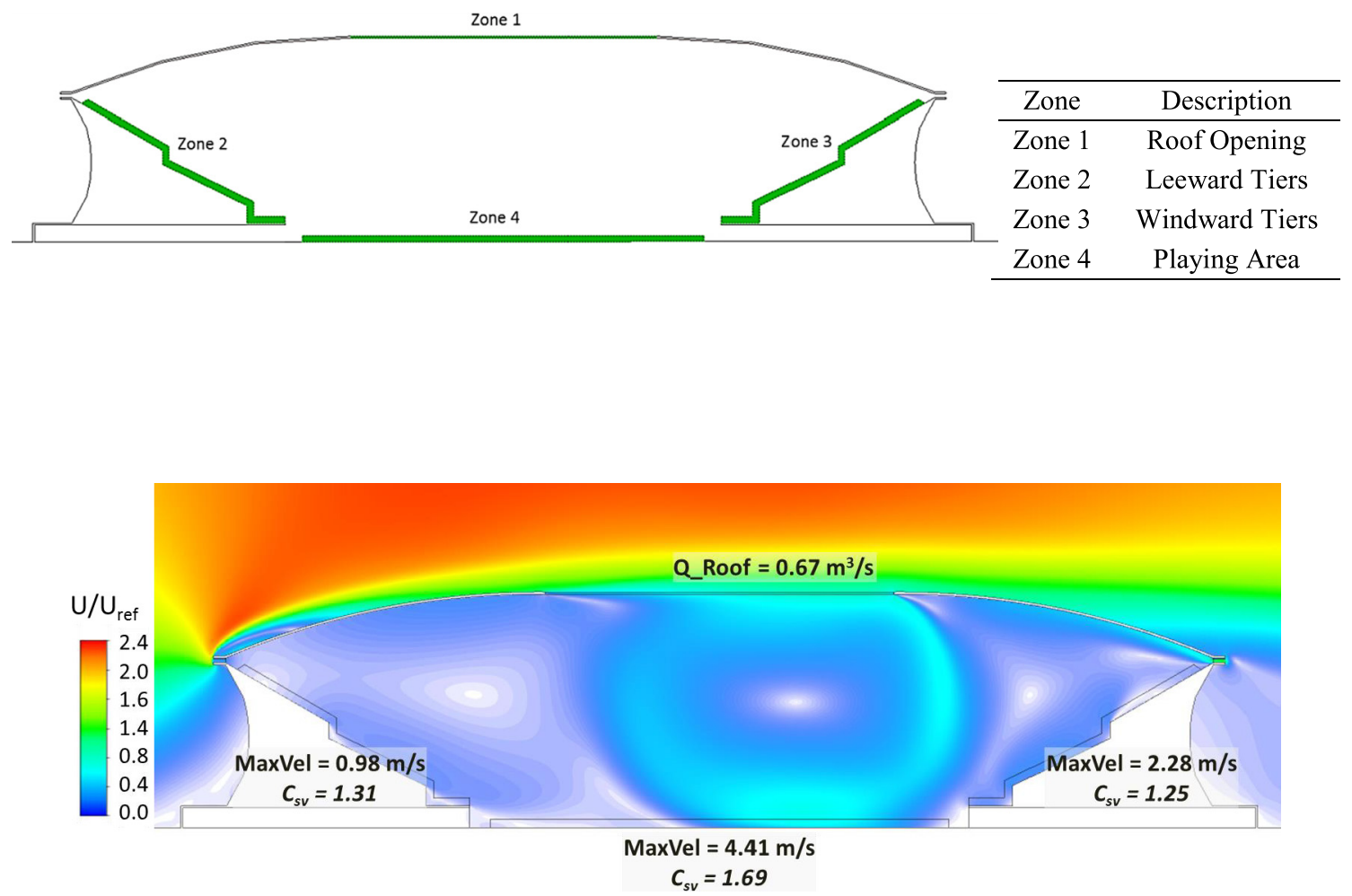

Fig. 8. Initial 2D stadium structure; illustration of velocity contours, values of homogeneity and maximum velocity speeds and roof flow rate.

ventilation openings located between the roof and the spectator terraces were selected as additional derived parameters. In order to control and enable the generation of the results, the areas of interest were separated from the entire fluid domain. As shown in Table 4, zones 2, 3 and 4 comprise an area equal to the zone length times $2 \mathrm{~m}$ on the perpendicular direction, to adequately cover the fluid area on human level. Zone 1 was $0.5 \mathrm{~m}$ thick, similar to roof thickness.

The flow homogeneity for ventilated areas was calculated based on the equation of the coefficient of spatial variation, presented in equation (4) [31]:

$$
C_{s v}=\sigma_{s}\left(\frac{V_{i}}{V_{r}}\right) / C_{v}
$$

where $C_{s v}$, coefficient of spatial variation, $C_{v}$, average velocity coefficient obtained by equation (5):

$$
C_{v}=1 \frac{1}{n} \sum_{i=1}^{n}\left(\frac{V_{i}}{V_{r}}\right)
$$

$\sigma_{s}\left(V_{i} / V_{r}\right)$ is the standard deviation of $C_{v}, V_{i}$ is the mean velocity at interior location $i(\mathrm{~m} / \mathrm{s}), V_{r}$ is the mean outdoor reference velocity at height $22 \mathrm{~m}(7 \mathrm{~m} / \mathrm{s})$, and $n$ is the number of nodes measured in the area of interest.
The coefficient of spatial variation, $C_{s v}$, was used as the indicator of the homogeneity at the zonal areas. Values of $C_{s v}$ larger than 1 imply distributions of high-variance, as opposed to values lower than 1 that indicate flow uniformity and velocity magnitudes of low-variance.

The ventilation flow rate was calculated based on equation (6), for a non-uniform flow of an incompressible fluid $(\rho=$ constant), in order to obtain a direct indicator of the flow direction, either incoming or outgoing flow stream to the stadium bowl.

$$
Q=\sum_{i=1}^{n} U_{i} A_{i}=\bar{U} A,
$$

where $A$ is the area of the opening configuration and $\bar{U}$ is the mean velocity in the perpendicular direction to the opening area $(A)$.

In Figure 8, the values of the output parameters for the initial stadium design are presented. The velocity values at the spectator tiers are lower than the velocity threshold of $3.6 \mathrm{~m} / \mathrm{s}$, as opposed to the playing field area. The calculated value of $4.41 \mathrm{~m} / \mathrm{s}$ is $18 \%$ higher than the recommended one that may result to players' performance disruption and compromise. The roof flow rate, with a value of $0.67 \mathrm{~m}^{3} / \mathrm{s}$ indicates that the air is leaving the stadium area, but this is due to the generated primary vortex that recirculates the air at the stadium interior. 
Table 5. Description of the input parameters and the defined constrains for spatial variance.

\begin{tabular}{cccc}
\hline $\begin{array}{c}\text { Input } \\
\text { Parameters }\end{array}$ & Name & Initial Value (m) & Constrains \\
\hline P1 & Roof_Radius_F & 200 & \multirow{2}{*}{$100 \mathrm{~m} \leq \mathbf{R} \leq 2000 \mathrm{~m}$} \\
P2 & Roof_Radius_B & 200 & $62 \mathrm{~m} \leq \mathbf{L} \leq 80 \mathrm{~m}$ \\
P3 & Roof_Length_F & 80 & \multirow{2}{*}{$0.9 \mathrm{~m} \leq \mathbf{H} \leq 20 \mathrm{~m}$} \\
\hline P5 & Roof_Length_B & 80 & 16 \\
& Roof_Height_B & 16 &
\end{tabular}

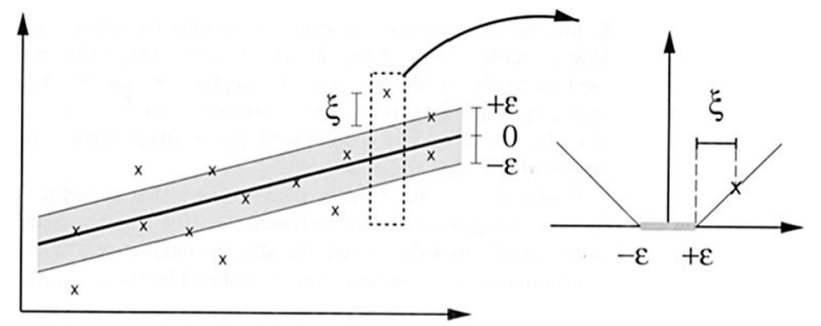

Fig. 9. Methodology followed by SVM hyperplanes to separate data in classes and minimise the distance of points falling out of the margins [35].

\subsection{Generation of design points}

The design space, within which different design points are evaluated, is defined as the design space that indicates the range of inputs' variability [32]. In the case of the stadium design, six input design parameters were selected, in order to control both symmetric and asymmetric transformations of the roof configuration, as presented in Table 5 . The main difference between the two case studies was that in symmetric case, the input parameters were reduced by three, since P2, P4 and P6 were derived parameters, equal to $\mathrm{P} 1, \mathrm{P} 3$ and $\mathrm{P} 5$, respectively. It is worth highlighting at this stage that the design constraints set for the input parameters were subjective, if not arbitrary, selected. The design exploration excluded the option of convex roof configuration, or a roof length that exposes the spectator tiers or partly covers the pitch area.

The selection of the appropriate scheme for the generation of the design points was performed with the Optimal Space-Filling design (OSF) with non-parametric regression model, because it can describe more accurately the nonlinear model parameters.

\subsubsection{OSF scheme}

The OSF scheme is usually employed in complex metamodelling techniques, such as the non-parametric regression, because it offers an extensive overview of the design space, by equidistributional selection of the input design parameters. The Max-Min Distance design type was selected, which maximises the minimum distance between any two points and it saves computational time. The number of the design points was based on full quadratic samples, by providing the number of the design points needed to generate a full quadratic model [33].

Based on the OSF scheme, 15 and 28 design points were generated in total, for the symmetric and asymmetric cases, respectively. The calculation of the design point responses (output parameters) was performed with sequential simulation runs, contributing to the most computational time-consuming part of the study, since convergence was achieved for every simulation run. The values of the output parameters will form the basis for the non-parametric regression model, as part of the RSM metamodel technique and the creation of the design space, where different roof configurations, regardless of the input value, may be examined against their responses.

\section{Response surface methodology}

The response surface methodology (RSM) technique employs mathematical and statistical tools to create approximation functions that can describe complex simulation data. Metamodel algorithms and mathematical relations are used to study the input and output values, their behaviour and interrelations, assisting model simplification, results' interpretation and performance of optimisation studies [34]. RSM determines the relationships among variables by performing regression analysis. 


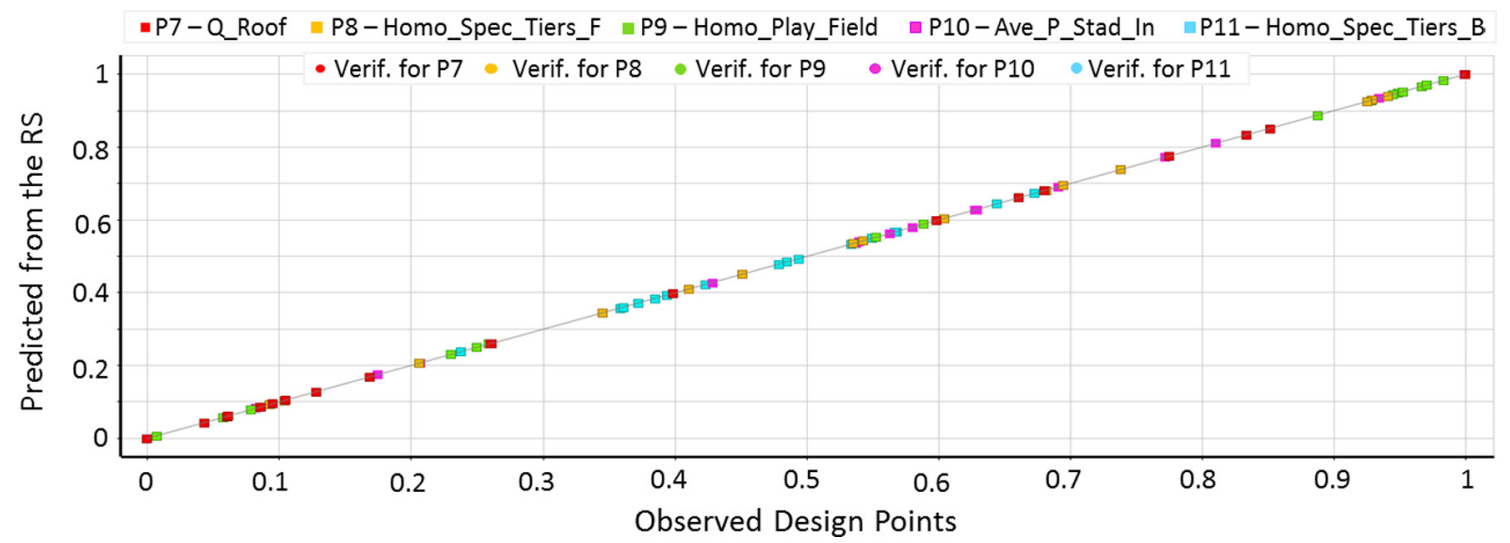

Fig. 10. Goodness of fit based on the non-parametric regression for the symmetric case.

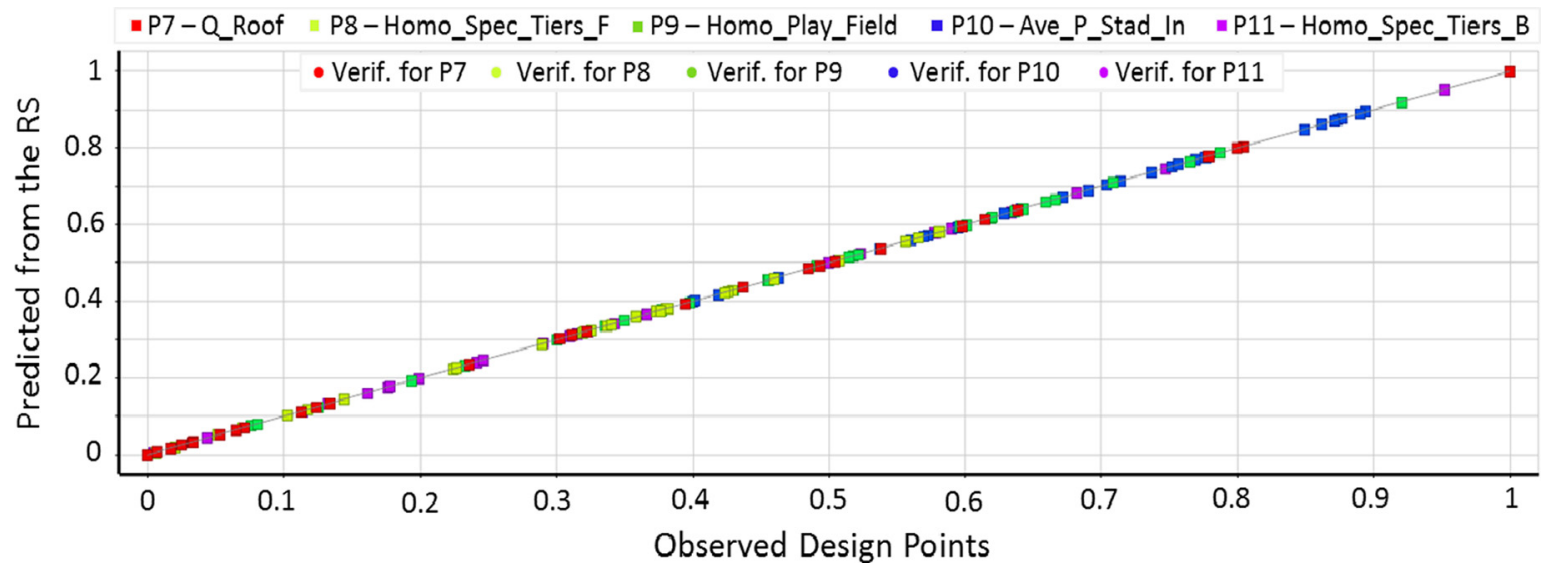

Fig. 11. Goodness of fit based on the non-parametric regression for the asymmetric case.

Depending on the number of the variables related to the problem, different algorithms may be applied, in order to determine the most accurate regression function that will describe the model. In the current study, for both symmetric and asymmetric roof alterations, the nonparametric regression meta-model algorithm was employed. The regression model enabled the prediction of the highly non-linear behaviour of the stadium's microclimate, with regard to the defined inputs.

\subsection{Non-parametric regression}

The non-parametric regression meta-model is based on the categorisation of noisy data using the Support Vector Machine (SVM) technique. SVM enables non-linear mapping of input-output data (or training data) on a higher-dimensional feature space by the aid of kernel maps. The data are split in non-overlapping classes by the aid of hyperplanes, aiming at the maximization of their inbetween margin. The hyperplanes are used to separate noisy data in groups, by using only those inputs, also known as support vectors that seem to sufficiently represent the outputs. At the same time, as illustrated in Figure 9, the hyperplanes are suitably positioned, so as to minimise the distance of the support vectors that fall outside the margin (slack variables $\xi$ ).
Nonparametric regression systems with non-linear responses and noisy results can be described mathematically by the following equations (7)-(9) [36]:

$$
f(x)=\langle w, x\rangle+b,
$$

where $f(x)$ is the response of interest, $\langle\cdot, \cdot\rangle$ is the dot product in $x$ and $w$ is a weight factor given by equation (8):

$$
w=\sum_{i=1}^{n}\left(\alpha_{i}^{*}-\alpha_{i}\right) \varphi\left(x_{i}\right) .
$$

Threshold $b$ can be described by equation (9):

$$
b=-\frac{1}{2}\left\langle w,\left(x_{r}+x_{s}\right)\right\rangle
$$

where $\alpha_{i}^{*}, \alpha_{i} \geq 0$ are Lagrange variables and $x_{r}$ and $x_{s}$ are support vectors.

The main advantage of non-parametric regression against the parametric one is that the function lacks of rigidity and can be adapted to the equivalent data without compromising form (parabolic, periodic, etc.), generating an inferred function [37]. 

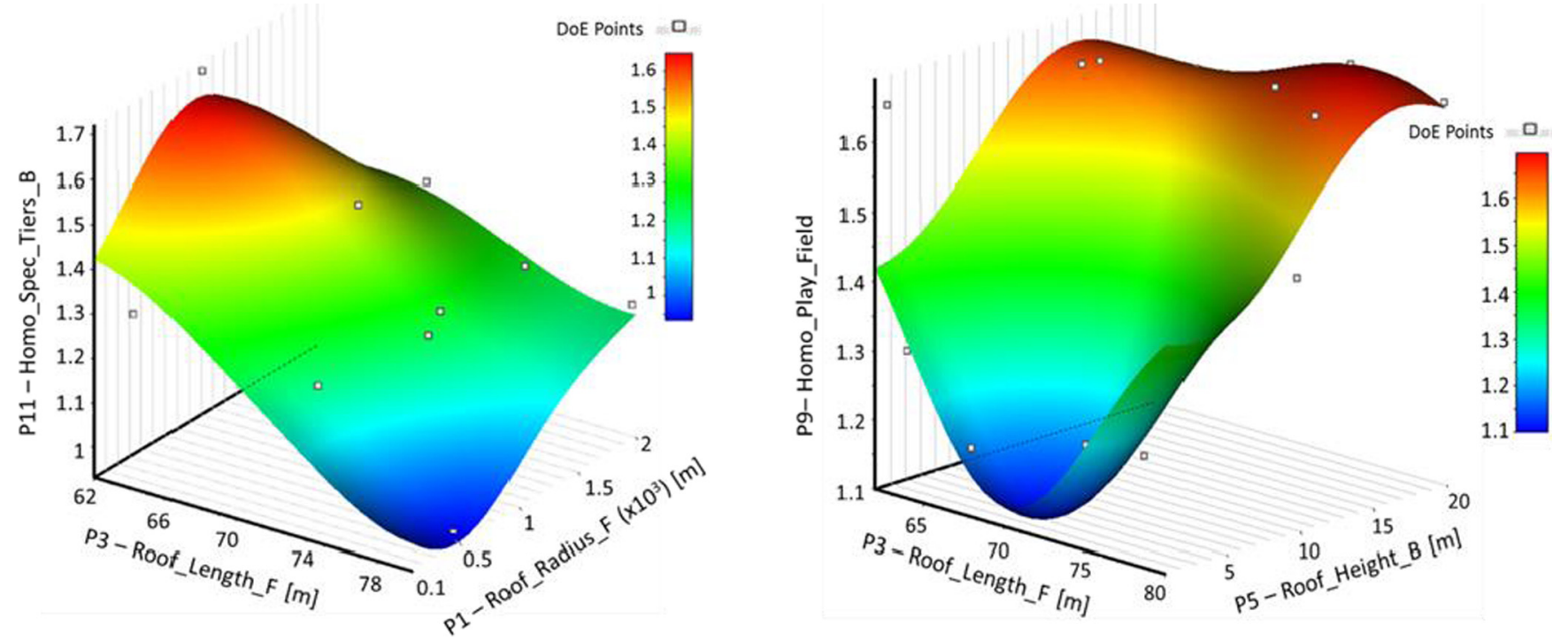

Fig. 12. Response surfaces for selective input-output parameters of the symmetric case.

Table 6. Optimisation objectives and constraints set for the output parameters.

\begin{tabular}{lll}
\hline Output parameter & Objective $\begin{array}{l}\text { Constraint } \\
\text { (lower bound) }\end{array}$ \\
\hline P7 - Q_Roof & - & Values $\geq 0 \mathrm{~m}^{3} / \mathrm{s}$ \\
P8 - Homo_Spec_Tiers_F & Minimise - \\
P9 - Homo_Play_Field & Minimise - \\
P10 - Ave_P_Stadium_In & Maximise - \\
P11 - Homo_Spec_Tiers_B & Minimise - \\
\hline
\end{tabular}

\subsubsection{Goodness of fit}

The goodness of fit gave a first insight into whether the data are adequately represented by the function. As shown in Figures 10 and 11, the predicted values from the response surface and the observed values form the design points perfectly fit the diagonal line, indicating a very good fit of the model.

A commonly used indicator, to show in what percentage a design output can be described by the response surface regression equation, is the coefficient of determination $\left(R^{2}\right)$. For linear regression analysis, the $R^{2}$ can be described by equation (10), and lies in the interval $[0,1]$.

$$
\begin{aligned}
R^{2} & =\frac{\text { Regression sum of squares }}{\text { Total sum of squares }} \\
& =\frac{\sum_{i=1}^{k}\left(\hat{y}_{i}-\bar{y}\right)^{2}}{\sum_{i=1}^{k}\left(y_{i}-\bar{y}\right)^{2}} .
\end{aligned}
$$

However, in case of non-parametric regression and nonlinear data, the integrity of the function cannot rely on the coefficients of determination $\left(R^{2}\right)$, because the more the parameters involved in the system, the higher the value of the regression sum of the squares will be, which might lead to a value of $R^{2}$ greater than 1.0 in some cases (see Eq. (10)) [38]. Indeed, in current case the values of $R^{2}$ for every design output were equal to 1.0 , as the software automatically truncates exceeding values to 1.0.

Since non-linear regression aims at minimizing the sum of squares of the points' distances from the fitting curve, the root mean square error (RMSE) can be considered a good estimator value, given by equation (11):

$$
\operatorname{RMSE}=\sqrt{\frac{1}{n} \sum_{i=1}^{n}\left(y_{i}-y_{i}\right)^{2}}
$$

The closer the value of RMSE is to zero, the better the fit. The values for the output values were varying from $2.2 \times 10^{-9}$ to $1.7 \times 10^{-4}$, indicating a very good fit and a regression function adequate to represent the model.

\subsection{Goal driven optimisation (GDO)}

The investigation of the optimum design solution presupposes the designation of the model objectives and constrains. The optimisation goal is to improve the stadium aero-comfort conditions, by providing homogeneity and low wind velocities at the zonal areas 2-4 (Tab. 4), and prevent the high flow rates via the roof opening. For the purpose of that, the optimisation targets set for both symmetric and asymmetric cases are presented in Table 6 .

The homogeneity (P8, P9 and P11) in all three occupied areas was set with a minimization goal. The low values of $C_{s v}$ (see Eq. (4)) will indicate lower flow heterogeneity and velocity variance. The average pressure at the stadium interior (P10) was deemed to be maximised, in order to prevent the large pressure differences between the external and the internal environment. The negative pressure at the stadium interior introduces large air streams flowing through the roof opening and the rear ventilation opening, disturbing the stadium's micro-climate. At the same time, a negative flow rate through the roof $(\mathrm{P} 7)$, calculated based 

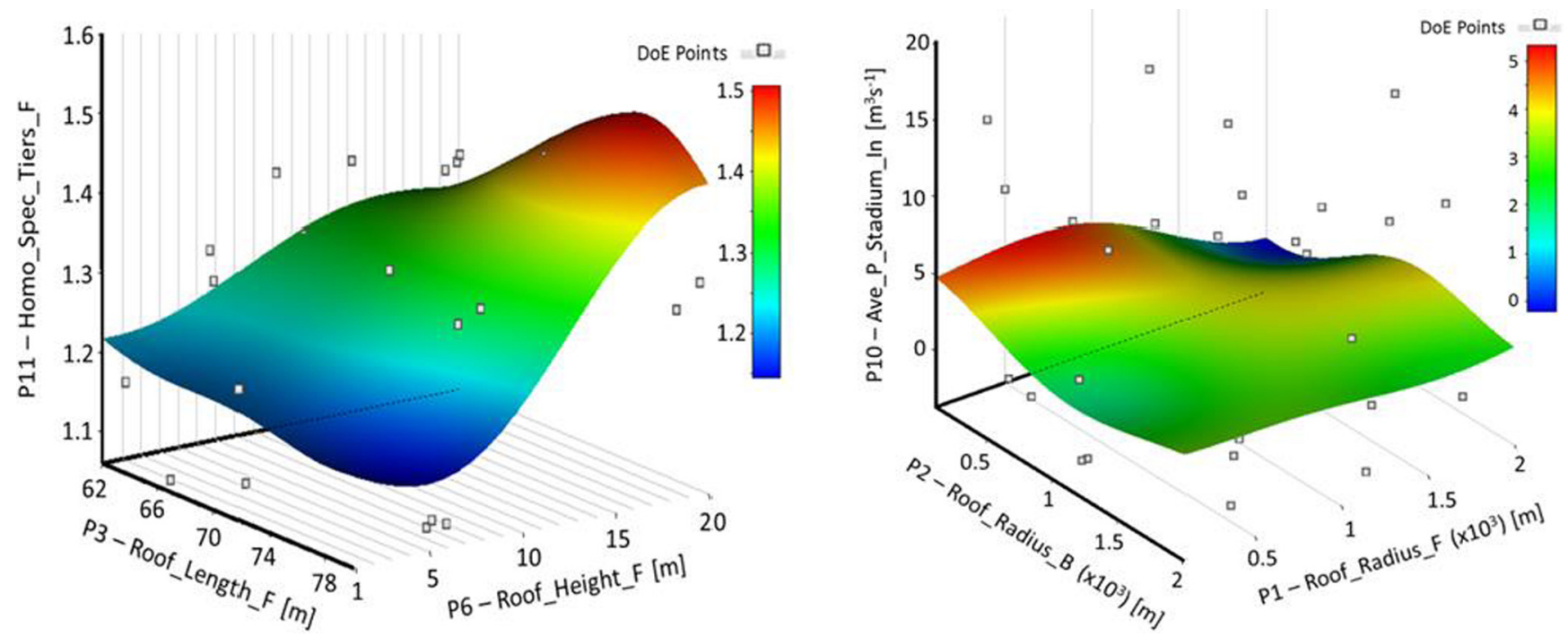

Fig. 13. Response surfaces for two sets of input-output parameters of the asymmetric case.

on equation (6) using the vertical component of velocity, indicates that more air is entering the stadium bowl than exiting. Thus, a lower bound constraint of $0 \mathrm{~m}^{3} / \mathrm{s}$ was added, in order to achieve air flow reduction.

The Multi-Objective Genetic Algorithm (MOGA) was used to conduct the multiobjective optimisation study. The MOGA belongs to the family of genetic algorithms (GA). By employing Pareto sets, a number of design solutions are determined, after assessing multiple populations of points. The algorithm performs an elitist non-dominated sorting according to the set objectives. The theoretical background can be found in $[39,41]$.

\section{Results and discussion}

\subsection{Response surfaces}

The response surface methodology gives a plethora of results, regarding the correlation of the input and output parameters, the sensitivity factors, indicating on what extent the input parameters influence the outputs, and the design spaces (or response surfaces), where any parameter can be evaluated against the responses.

Three-dimensional graphs of the responses were generated, enabling the investigation of the effect that two inputs have on a single output. Due to the multidimensional character of the system, few response surfaces are selectively presented that show the complexity of the model. For the symmetric case study, the response surfaces of the flow homogeneity on the back spectator tiers (P11) and the play field area (P9) are presented in Figure 12, regarding the alterations in the roof length and radius (P1, P3) and the roof length and height (P3, P5), respectively. On linear correlations between the parameters, the response surface would fit perfectly the design points. However, this is not the case in the current design problem. The system is highly non-linear and the algorithm adopted, used weighting factors to create a function of high accuracy that enables the prediction of any response within the input design space.
Based on the local sensitivities, by increasing the roof height, the roof flow rate and the homogeneity in the front spectator tiers and playing field area are decreased, as opposed to the average pressure and the homogeneity on the back spectator tiers that are increased. Moreover, the average pressure (P8) is increased by increasing the roof radius, and the homogeneity on the back spectator tiers is also increased by increasing the roof length.

Similar results were obtained for the asymmetric design case. The even larger number of independent parameters, led to a multivariable system, responses of which can be found in Figure 13. In this case, the front roof height (P6) seems to be determinant for the majority of outputs. The increasing value of P6 leads to reduced roof flow rate and poor homogeneity on the front spectator tiers, as opposed to the average pressure and the homogeneity on the back spectator tiers, which are increased. Additional to these, as the back roof height increases, the roof flow rate is increased, and the average pressure and the playing field homogeneity are decreased.

\subsection{Optimisation results}

The MOGA meta-model algorithm generated in total six candidate points that performed better based on the defined set of objectives. Table 7 shows the optimisation results generated for the symmetric roof modifications. The geometrical patterns look quite similar for all three candidate points. More specifically, the roof height was reduced by a maximum of $70 \%$ and a minimum of $57 \%$ for the $\mathrm{CP} 3$ and $\mathrm{CP} 1$ respectively. The roof radius of $\mathrm{CP} 3$ was increased to more than $565 \%$, resulting in an almost flat roof configuration. Finally, the length was reduced between $68.8 \mathrm{~m}$ and $71.1 \mathrm{~m}$, providing full protection to the spectator terraces and exposing adequately the pitch area.

The simulated velocity contours indicate how the roof modification may alter dramatically the environment at the stadium interior. The centre of the primary vortex was relocated vertically upwards, resulting in positive flow rate values. Contrariwise, the creation of a new recircula- 
Table 7. Candidate points generated based on MOGA algorithm for symmetric case.

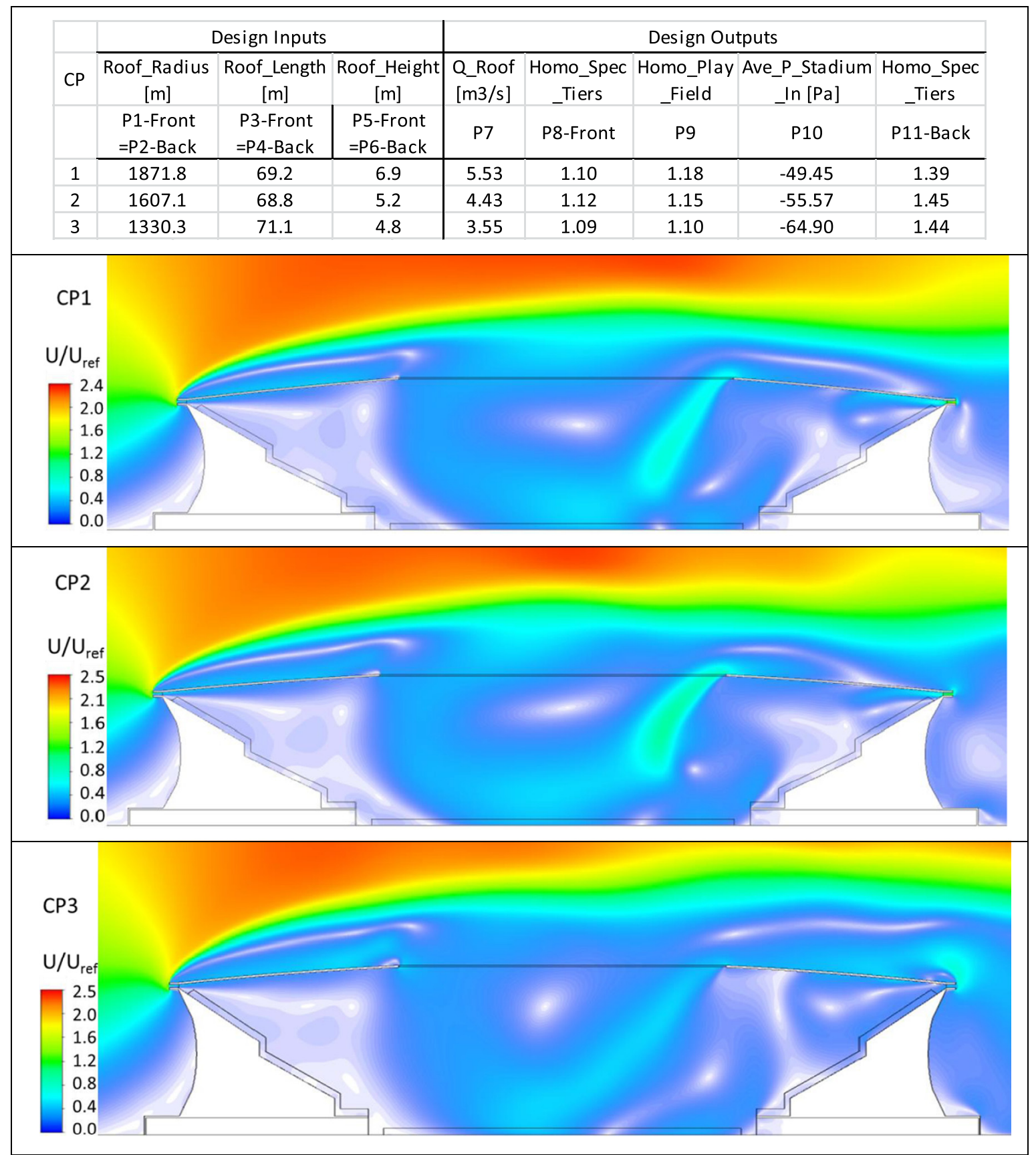

tion zone above the rear roof geometry is now considered responsible for the air entering the stadium bowl with high-speed values that are weakened before reaching pitch level. The secondary vortex located at the back spectator tiers was moved towards the pitch level. The air entering through the back ventilation opening resulted in the generation of another vortex limited to the top spectator tiers, for $\mathrm{CP} 1$ and $\mathrm{CP} 3$. For the CP1, the same vortex is of lower intensity and spatial extension. In any design solution, these vortices promoted flow heterogeneity, resulting to higher values of $C_{s v}$. The change in roof height and radius resulted in reverse air direction on the front ventilation opening. The impinging air enters the stadium and partly feeds the present secondary vortex and partly enhances the primary vortex to exit the stadium area.

In Table 8, the candidate points for the asymmetric roof optimisation are presented. In order to avoid the air entrance at the stadium interior through the roof opening, 
Table 8. Candidate points generated based on MOGA algorithm for asymmetric case.

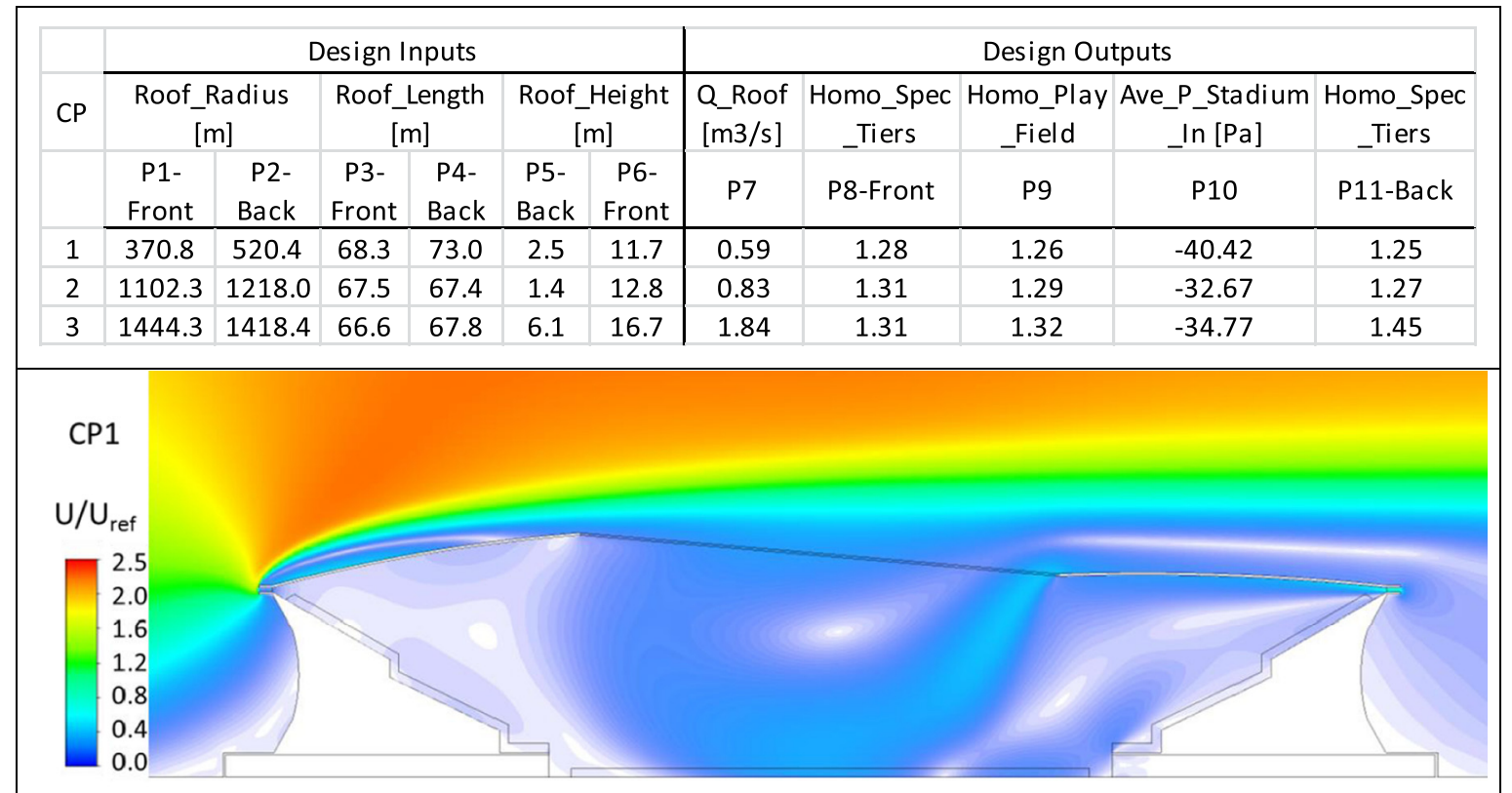
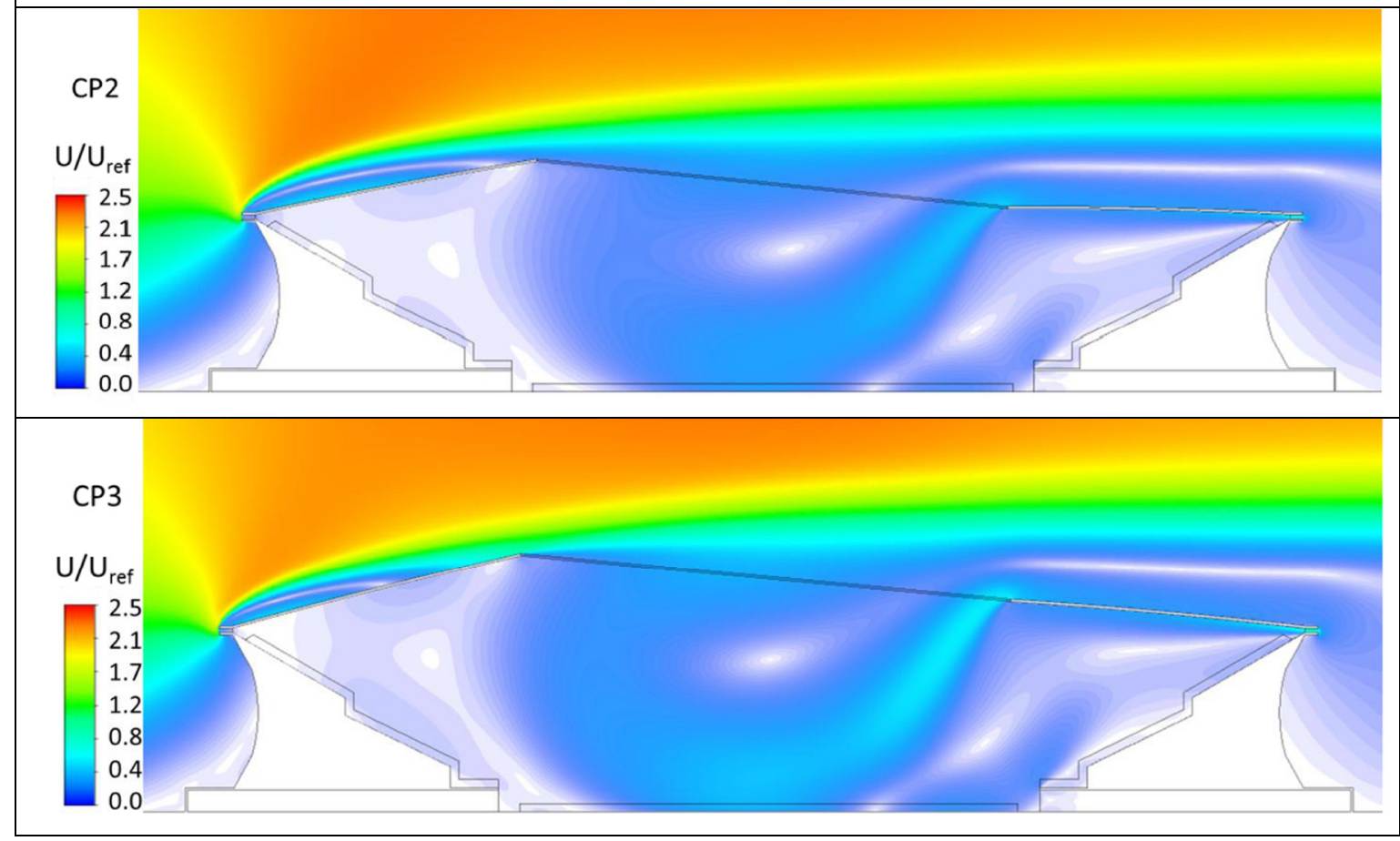

the back roof height (P4) was decreased by a maximum of $91.25 \%$ for CP2 and a minimum of $61.87 \%$ for CP3. The difference in height allows the air to pass above the roof construction, without entering the stadium interior. The recirculation zone that is created behind the stadium structure drives the air through the rear ventilation opening and at the same time contributes to the generation of a recirculation zone above the back roof part. In this case, two factors are responsible for the generation of the primary vortex. The first one is due to the aforementioned recirculation area at the top of the roof surface and the second one is due to the incoming air through the rear ventilation opening. Similarly, as in symmetric case, the secondary vortex in the back spectator tiers is relocated towards the pitch area, providing lower velocity values at zone 3 , but slightly higher heterogeneity. The flow homogeneity in the pitch area has been increased by a minimum of $132 \%$, whereas in the front spectator tiers 


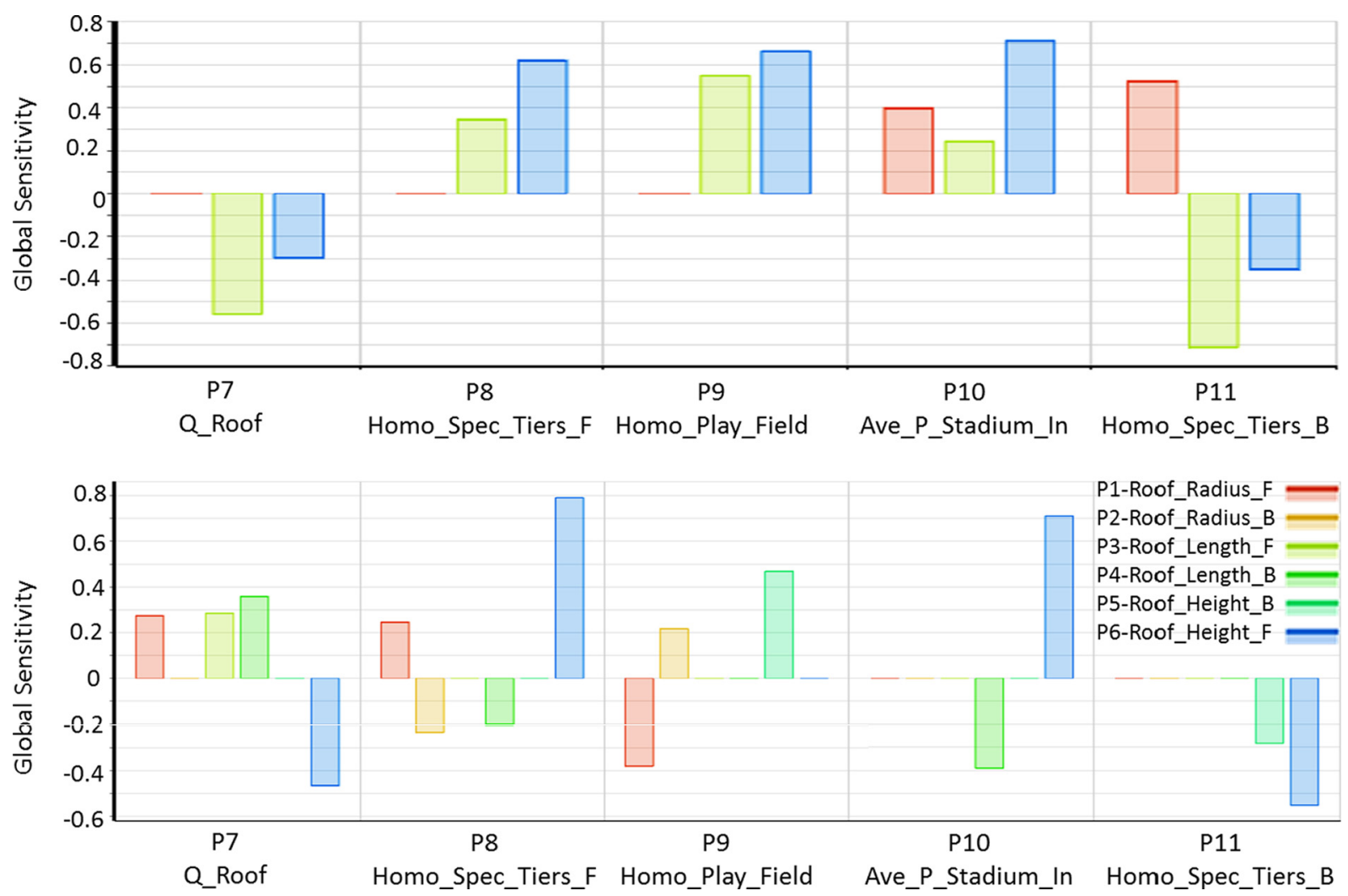

Fig. 14. Global sensitivity chart of input-output parameters for the symmetric (top) and asymmetric (bottom) case.

remained relatively stable over the same values. Finally, the front secondary vortex is spatially limited, by the prevailing primary one and with considerable lower velocity magnitude intensity.

\subsection{GDO sensitivities}

Using correlation analysis, the global sensitivities over the output parameters were generated, by evaluating the entire design space. Figure 14 (top) shows the global sensitivities for the symmetric case study. According to the results, the roof height and length have great impact (either positive or negative) in all the design responses as compared to roof radius. Positive sensitivity denotes that as the value of the design parameter increases, the value of the design response will increase too. Negative sensitivity implies that increasing the value of the design parameter, the response value will be decreased. The roof radius appears to have positive sensitivity in both the average pressure at the stadium interior and the values of homogeneity at the back spectator tiers. It also has negligible impact on the parameters P7, P8 and P9.

In case of the asymmetric roof configuration (Fig. 14, bottom), the front roof height (P6) seems to have the greatest impact both positive and negative in almost all of the output parameters. Considering wind is unidirectional, it is expected that P6 will determine mostly the flow at the stadium interior. In case of the homogeneity in the playing field area, P6 seems to have no impact. This can be explained graphically by the figures in Table 8 , where a recirculation zone is created on top of the back roof part and drives the air at the stadium interior, and towards the pitch area.

\subsection{GDO verification}

The verification of the candidate points for optimum aerocomfort conditions at the stadium micro-environment was performed by CFD simulation runs. Figure 15 compares the results of the candidate points obtained from the GDO and the CFD simulations for the symmetric roof modification. The values of homogeneity in the zonal areas 2, 3 and 4 (P8, P9 and P11) show good prediction performance, with a maximum error of $16.35 \%$ associated with the homogeneity values at the back spectator tiers (P11) for the CP2, followed by an error of $11.04 \%$ for the same output parameter for the CP1. The development of vortices at the windward spectator tiers is susceptible to disturbances caused by the induced wind through the ventilation openings, and thus the computation of velocity magnitudes is highly sensitive. The flow rate values $(\mathrm{P} 7)$ were predicted with a maximum absolute deviation of $1.84 \mathrm{~m}^{3} / \mathrm{s}$ for the CP2. Considering the roof length opening of $116.5 \mathrm{~m}$ and the presence of the central primary vortex, the difference in flow rate can be consider substantially low. The largest errors were observed for the average pressure (P10) for all three candidate points. With the maximum absolute error to be as high as $29.20 \mathrm{~Pa}$, the pressure output could not be considered an accurate parameter to be 

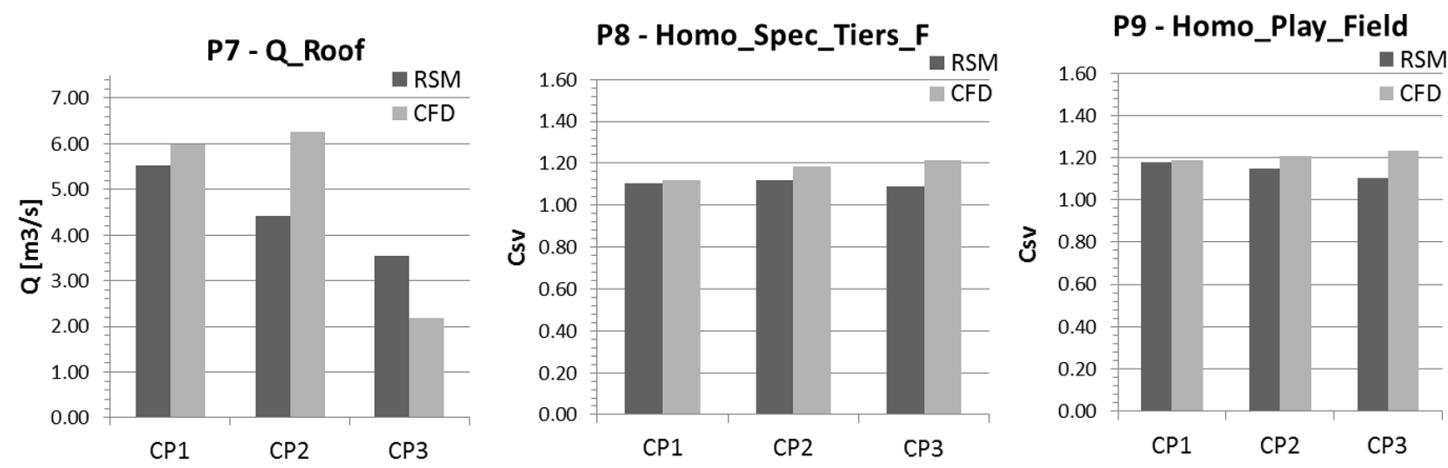

P10 - Ave_P_Stadium_In
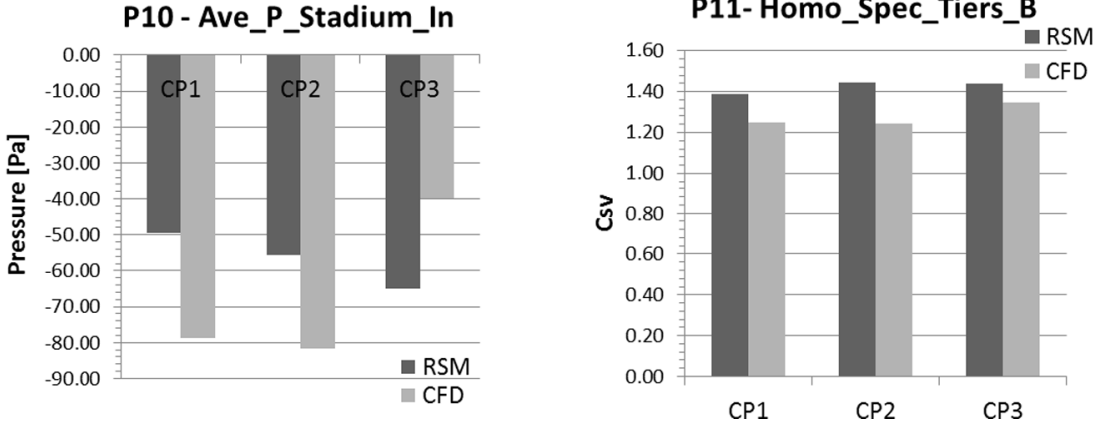

Fig. 15. RSM vs. CFD results for verification for the three candidate points for the symmetric case.
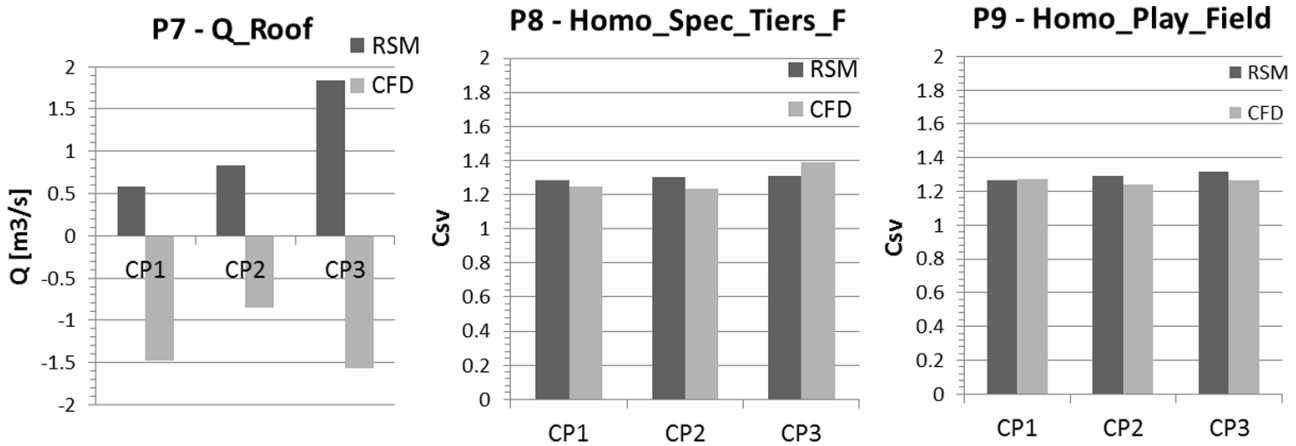

P10 - Ave_P_Stadium_In

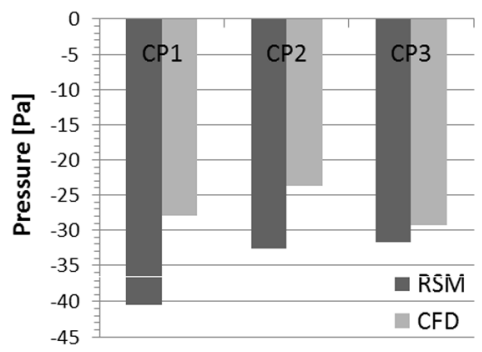

P11- Homo_Spec_Tiers_B

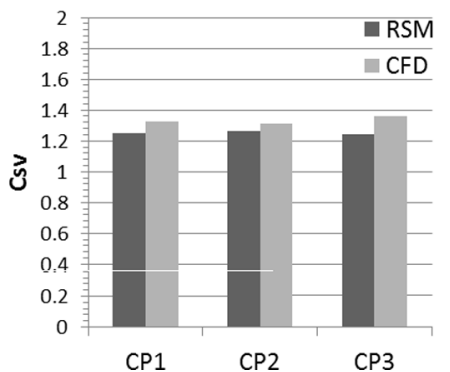

Fig. 16. RSM vs. CFD results for verification of the three candidate points for the asymmetric case.

considered for the selection of the optimum solution. The generated recirculation zones and wind vortices make the average pressure imprecise and difficult to predict.

In Figure 16, the comparison of the results for the asymmetric roof indicated a GDO of higher performance. The homogeneity values were predicted with a maximum error of $8.65 \%$ for the CP3. The maximum absolute deviations of the average pressure (P10) and the flow rate (P7) were equal to $12.4 \mathrm{~Pa}$ for the $\mathrm{CP} 1$ and $3.4 \mathrm{~m}^{3} / \mathrm{s}$ for the CP3, respectively.

The verification study has highlighted the inaccuracies in predicting some output parameters, such as the average pressure (P10) and the flow rate (P7), causing some sort of compromise in the optimisation process. Regarding the 

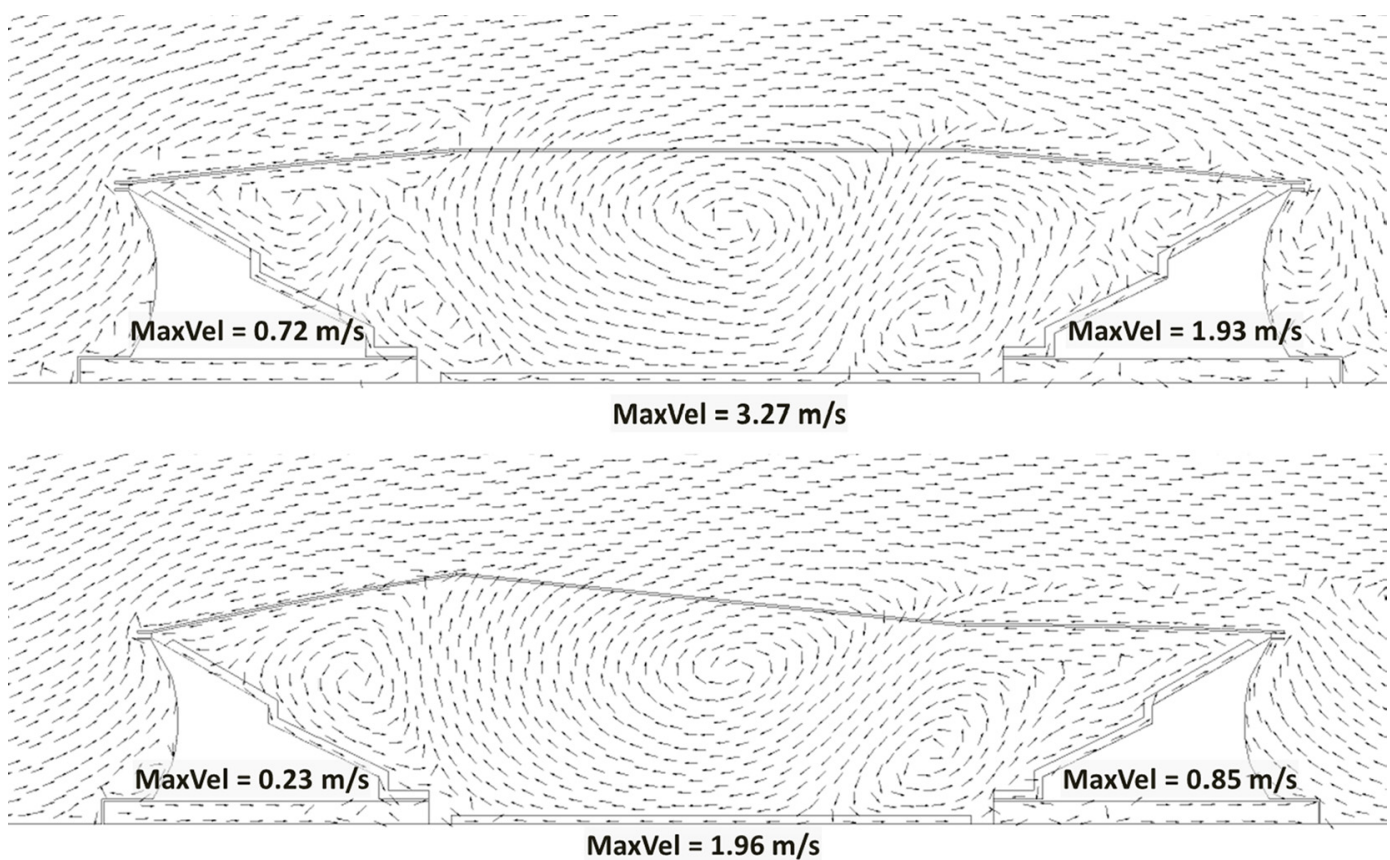

Fig. 17. Normalised velocity vectors and maximum zonal velocity magnitude for the optimum symmetric (top) and asymmetric (bottom) case.

number of variables involved and the complexity of the model, the verification enabled the assessment of the best roof configuration among the candidates, revealing the global optimum design solutions. For the symmetric case study, the CP1 had the best performance according to the CFD simulation results. Likewise, the CP2 in the asymmetric roof configuration had the less deviation in all parameters against the CFD results.

\section{Interpretation of results}

The verified optimum solutions are further discussed in terms of maximum velocity magnitudes and internal flow distribution. Figure 17 presents the normalised velocity vectors for the $\mathrm{CP} 1$ of the symmetric and the $\mathrm{CP} 2$ of the asymmetric roof configuration.

The roof optimisation contributed to a total reduction of $26.5 \%, 15.4 \%$ and $25.9 \%$ for the maximum velocities in the front, back spectator tiers and the pitch area for the symmetric case and $76.5 \%, 62.7 \%$ and $55.6 \%$ for the asymmetric case, respectively. Thus, the maximum allowed mechanical threshold of $3.6 \mathrm{~m} / \mathrm{s}$ for stadia was satisfied for both designs. Figure 18 shows a graphical comparison of the dimensionless horizontal component of velocity for the front and back spectator tiers before and after optimisation. It can be concluded that the microenvironment at the spectator terraces is substantially less determined by the primary vortex and consequently by the induced air through the roof opening.

\subsection{Limitations and suggestions for future studies}

The two-dimensional study poses certain limitations regarding the lack of representing adequately 3D flow characteristics, including turbulent dispersion, buoyancy effects and capture of the separation zones. However, the qualitative study gives a further insight on the design optimisation of the stadium roof geometry for improved aero-comfort conditions in different areas in the stadium. The optimisation method and results presented in this work aim to form the basis for a future study of a 3D stadium design. The definition of design constraints and objectives limits the exploration of solutions of global optimality. The study was additionally formed under subjective selection of input and output parameters. Thus, different design solutions may be obtained under different sets of parameters and objectives.

\section{Conclusion}

In the present study, a qualitative optimisation study of a 2D stadium roof configuration was performed, with the intention to improve the wind comfort conditions at the stadium bowl. Based on coupled CFD and RSM techniques, the selected design parameters were assessed with reduced computational time and cost, as opposed to traditional deterministic studies. The initial stadium design was simulated using the FLUENT code and validated against the study of Persoon et al. [5] for airflow distribution in football stadia. The investigation of different roof configurations, based on variable height, length and radius, was conducted using design experimentation. Both symmetrical and asymmetrical modifications were assessed against the micro-climate conditions developed at the stadium interior, and 6 candidate points were generated in total. CP1 for the symmetric and CP2 asymmetric case were proven to have the best performance against the CFD-based verification. 



Fig. 18. Comparison of the dimensionless horizontal component of velocity for symmetric (top) and asymmetric (bottom) case, before (red line) and after (green line) optimisation.

The results revealed that the optimal symmetric roof design may contribute to a maximum velocity reduction of up to $26.5 \%$ for the front spectator tiers, if the roof height is reduced by $57 \%$ and the roof radius increased by $835 \%$, over the initial one. In the asymmetric roof configuration, the front roof part seems to be the most crucial for the airflow distribution. With an asymmetric roof difference of $11.4 \mathrm{~m}$ the maximum reduction observed for the maximum velocity was equal to $75 \%$. For both designs, wind comfort was achieved that complies with the wind thresholds set for stadiums. Considering the limitations of conducting a twodimensional study, the optimisation method and qualitative results presented in this work will form the preliminary basis for future optimisation study of a complex $3 \mathrm{D}$ stadium structure.

This paper was made possible by a NPRP grant (6-461-2-188) from the Qatar National Research Fund (A member of the Qatar Foundation). The statements made herein are solely the responsibility of the authors.

\section{References}

1. A. Parr, M. Zaretsky, New directions in sustainable design (Routledge, London, 2011)

2. K. Lomas, H. Eppel, M. Cook, J. Mardaljevic, Ventilation and thermal performance of design options for Stadium Australia, in: The 5th International IBPSA Conference [online] (International Building Performance Simulation Association, Prague, 1997), Available at: https://dspace. lboro.ac.uk/2134/12393

3. D. Fiala, K.J. Lomas, 1999, Application of a computer model predicting human thermal responses to the design of sports stadia, in: CIBSE'99, Conference Proc., Harrogate, UK, pp. 492-499

4. J. Bouyer, J. Vinet, P. Delpech, S. Carre, Thermal comfort assessment in semi-outdoor environments: application to comfort study in stadia, J. Wind Eng. Ind. Aerodyn. 95, 963 (2007)

5. J. Persoon, T. van Hooff, B. Blocken, J. Carmeliet, M. de Wit, On the impact of roof geometry on rain shelter in football stadia, J. Wind Eng. Ind. Aerodyn. 96, 1274 (2008) 
6. A. Szucs, S. Moreau, F. Allard, Aspects of stadium design for warm climates, Build. Environ. 44, 1206 (2009)

7. T. van Hooff, B. Blocken, M. van Harten, 3D CFD simulations of wind flow and wind-driven rain shelter in sports stadia: influence of stadium geometry, Build. Environ. 46, $22(2011)$

8. P. Biagini, C. Borri, L. Facchini, Wind response of large roofs of stadions and arena, J. Wind Eng. Ind. Aerodyn. 95, 871 (2007)

9. T. van Hooff, B. Blocken, Full-scale measurements of indoor environmental conditions and natural ventilation in a large semi-enclosed stadium: possibilities and limitations for CFD validation, J. Wind Eng. Ind. Aerodyn. 104, 330 (2012)

10. B. Blocken, J. Persoon, Pedestrian wind comfort around a large football stadium in an urban environment: CFD simulation, validation and application of the new Dutch wind nuisance standard, J. Wind Eng. Ind. Aerodyn. 97, 255 (2009)

11. T. van Hooff, B. Blocken, Coupled urban wind flow and indoor natural ventilation modelling on a high-resolution grid: a case study for the Amsterdam ArenA stadium, Environ. Model. Softw. 25, 51 (2010)

12. T. van Hooff, B. Blocken, On the effect of wind direction and urban surroundings on natural ventilation of a large semienclosed stadium, Comput. Fluids 39, 1146 (2010)

13. A. Stamou, I. Katsiris, A. Schaelin, Evaluation of thermal comfort in Galatsi Arena of the Olympics "Athens 2004" using a CFD model, Appl. Therm. Eng. 28, 1206 (2008)

14. M. Ucuncu, D. Woolf, M. Zikri, Thermal comfort of spectators in stadia built in hot climates, paper presented at Adapting to Change: New Thinking on Comfort, Windsor, UK, 9-11April (Network for Comfort and Energy Use in Buildings, London, 2010), p. 8

15. A. Matzarakis, D. Fröhlich, Sport events and climate for visitors - the case of FIFA World Cup in Qatar 2022, Int. J. Biometeorol. 59, 481 (2014)

16. T. Nishioka, K. Ohtaka, N. Hashimoto, H. Onojima, Measurement and evaluation of the indoor thermal environment in a large domed stadium, Energy Build. 32, 217 (2000)

17. P. Sofotasiou, B. Hughes, J. Calautit, Qatar 2022: Facing the FIFA World Cup climatic and legacy challenges, Sustain. Cities Soc. 14, 16 (2015)

18. B. Blocken, 50 years of Computational Wind Engineering: Past, present and future, J. Wind Eng. Ind. Aerodyn. 129, 69 (2014)

19. P. Sofotasiou, J. Calautit, B. Hughes, D. O'Connor, Towards an integrated computational method to determine internal spaces for optimum environmental conditions, Comput. Fluids 127, 146 (2016)

20. FIFA, Football Stadiums: Technical recommendations and requirements, 5th edition [e-book] (FIFA, 2011), p. 108, 283

21. F. Lien, E. Yee, Y. Cheng, Simulation of mean flow and turbulence over a 2D building array using high-resolution CFD and a distributed drag force approach, J. Wind Eng. Ind. Aerodyn. 92, 117 (2004)

22. X. Xiaomin, H. Zhen, W. Jiasong, The impact of urban street layout on local atmospheric environment, Build. Environ. 41, 1352 (2006)
23. Y. Huang, X. Hu, N. Zeng, Impact of wedge-shaped roofs on airflow and pollutant dispersion inside urban street canyons, Build. Environ. 44, 2335 (2009)

24. L. Yik, S. Salim, A. Chan, C. Cheong, CFD study of flow over parallel ridges with varying height and spacing, in: World Congress on Engineering, London (2010), Available at: http://www.iaeng.org/publication/ WCE2010/WCE2010_pp1206-1211.pdf

25. F. Joerg, Recommendations of the COST action C14 on the use of CFD in predicting pedestrian wind environment, Fourth Int. Symp. Comput. Wind Eng. 2006, 529 (2006)

26. P. Richards, R. Hoxey, Appropriate boundary conditions for computational wind engineering models using the $\mathrm{k}-\varepsilon$ turbulence model, J. Wind Eng. Ind. Aerodyn. 46-47, 145 (1993)

27. D. Hargreaves, N. Wright, On the use of the $\mathrm{k}-\varepsilon$ model in commercial CFD software to model the neutral atmospheric boundary layer, J. Wind Eng. Ind. Aerodyn. 95, 355 (2007)

28. J. Wieringa, Updating the Davenport roughness classification, J. Wind Eng. Ind. Aerodyn. 41, 357 (1992)

29. X. Shen, G. Zhang, B. Bjerg, Investigation of response surface methodology for modelling ventilation rate of a naturally ventilated building, Build. Environ. 54, 174 (2012)

30. A. Szucs, S. Moreau, F. Allard, Spectators' aerothermal comfort assessment method in stadia, Build. Environ. 42, 2227 (2007)

31. J. Kindangen, G. Krauss, P. Depecker, Effects of roof shapes on wind-induced air motion inside buildings, Build. Environ. 32, 1 (1997)

32. M. Cavazzuti, Optimization methods (Springer, Berlin, 2013)

33. ANSYS, Design Exploration Release 12.1, no. November (ANSYS, Inc, South-pointe 275 Technology Drive Canonsburg, PA 15317, USA, 2009)

34. L. Friedman, The simulation metamodel (Springer US, Boston, MA, 1996)

35. B. Schölkopf, C. Burges, A. Smola, Advances in kernel methods (MIT Press, Cambridge, MA, 1999), p. 11

36. V. Vapnik, The nature of statistical learning theory (Springer, New York, 2000)

37. M. Wand, M. Jones, Kernel smoothing (Chapman \& Hall, London, 1995)

38. T. Kvålseth, Note on the $R^{2}$ measure of goodness of fit for nonlinear models, Bull. Psychon. Soc. 21, 79 (1983)

39. K. Deb, A. Pratap, S. Agarwal, T. Meyarivan, A fast and elitist multiobjective genetic algorithm: NSGA-II, IEEE Trans. Evol. Comput. 6, 182 (2002)

40. C. Foncesa, P. Fleming, Genetic algorithms for multiobjective optimization: formulation, discussion and generalization, in: Fifth International Conference on Genetic Algorithms (Morgan Kauffman, San Mateo, CA), 416 (1993)

41. S.F. Fatima, H.N. Chaudhry, Steady-state CFD modelling and experimental analysis of the local microclimate in Dubai (UAE), Sust. Build. 2, 5 (2017)

42. F. Calcerano, C. Cecchini, L. Martinelli, Numerical analysis of passive strategies for energy retrofit of existing buildings in Mediterranean climate: thermal mass and natural ventilation combination, Sust. Build. 2, 4 (2017)

Cite this article as: P. Sofotasiou, B. Hughes and S.A. Ghani: CFD optimisation of a stadium roof geometry: a qualitative study to improve the wind microenvironment. Sust. Build. 2, 8 (2017). 